\title{
Computational thermomechanics of crystalline rock, Part I: A combined multi-phase-field/crystal plasticity approach for single crystal simulations
}

\author{
SeonHong Na, WaiChing Sun* \\ Department of Civil Engineering and Engineering Mechanics, Fu Foundation School of Engineering and Applied Science, Columbia University \\ in the City of New York, 614 SW Mudd, Mail Code: 4709, New York, NY 10027, United States
}

Received 13 September 2017; received in revised form 24 November 2017; accepted 18 December 2017

Available online 5 January 2018

\begin{abstract}
Rock salt is one of the major materials used for nuclear waste geological disposal. The desired characteristics of rock salt, i.e., high thermal conductivity, low permeability, and self-healing are highly related to its crystalline microstructure. Conventionally, this microstructural effect is often incorporated phenomenologically in macroscopic damage models. Nevertheless, the thermomechanical behavior of a crystalline material is dictated by the nature of crystal lattice and micromechanics (i.e., the slip-system). This paper presents a model proposed to examine these fundamental mechanisms at the grain scale level. We employ a crystal plasticity framework in which single-crystal halite is modeled as a face-centered cubic (FCC) structure with the secondary atoms in its octahedral holes, where a pair of $\mathrm{Na}^{+}$and $\mathrm{Cl}^{-}$ions forms the bond basis. Utilizing the crystal plasticity framework, we capture the existence of an elastic region in the stress space and the sequence of slip system activation of single-crystal halite under different temperature ranges. To capture the anisotropic nature of the intragranular fracture, we couple a crystal plasticity model with a multi-phase-field formulation that does not require high-order terms for the phase field. Numerical examples demonstrate that the proposed model is able to capture the anisotropy of inelastic and damage behavior under various loading rates and temperature conditions.
\end{abstract}

(C) 2017 Elsevier B.V. All rights reserved.

Keywords: Halite; Rock salt; Thermo-mechanics; Crystal plasticity; Anisotropic damage

\section{Introduction}

The demands for safe and permanent disposal of nuclear waste in geologic formations date back over decades. Natural rock salt, found in domal and bedded formations and the re-consolidated counterpart formed in a high-pressure and high-temperature environment, has been used for geological repositories of nuclear waste disposal in the United States and Germany [1]. Two operating facilities include the Waste Isolation Pilot Plant (WIPP) in Carlsbad (New

\footnotetext{
* Corresponding author.

E-mail address: wsun@ columbia.edu (W. Sun).
} 


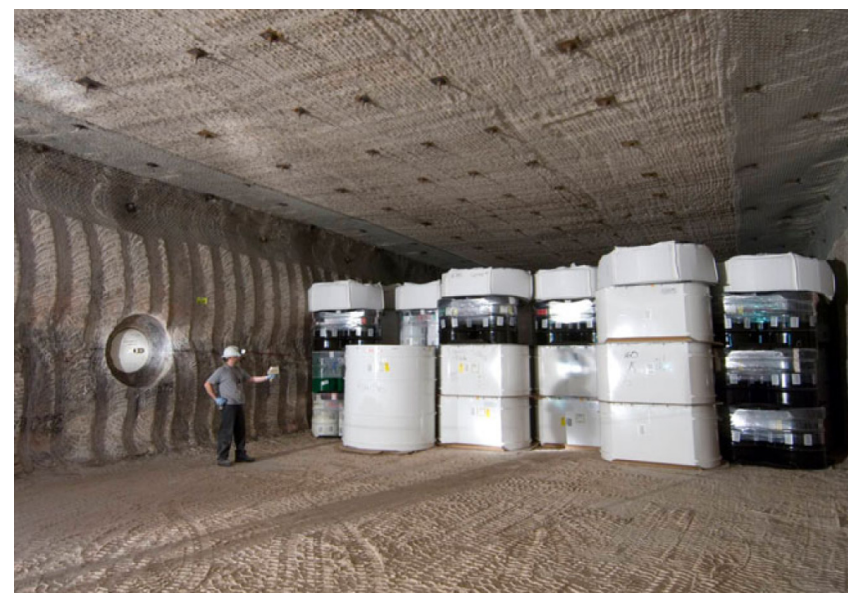

Fig. 1. Disposal operations for Transuranic (TRU) waste at the Waste Isolation Pilot Plant (WIPP). Source: Reproduced from Hansen and Leigh [6].

Mexico, USA), and the Endlager für radioaktive Abfälle Morsleben (ERAM) site in Morsleben, Germany [2]. (See Fig. 1.)

The decision to use salt formation for storage and disposal of radioactive wastes is attributed to its desirable thermo-hydro-mechanical-chemical characteristics, i.e., (1) high thermal conductivity, (2) low permeability, (3) selfhealing mechanism, and (4) biologically inactivity of rock salt (as compared with clay). Firstly, the heat generated from nuclear wastes can be dissipated to the surrounding area much faster in salt than in other materials since the host salt rock exhibits high thermal conductivity [3]. In addition, the permeability of rock salt is sufficiently low that it is often idealized as impermeable. Therefore, it may function as a secured barrier for radioactive wastes [4]. Finally, the creeping property of salt enables microcracks or damage under mechanical load to be self-sealed, which may also naturally guarantee the necessary geological barrier function (e.g., Chan et al. [5], von Berlepsch and Haverkamp [2]).

Decades of experimental investigations for rock salt provided insight on its mechanisms of brine migration, vapor transport, and related solution-precipitation creeps under nonisothermal conditions (e.g., Bradshaw and Sanchez [7], Nowak and McTigue [8], Beauheim et al. [9], Schléder et al. [10], Hansen et al. [11], Kuhlman and Malama [1]). However, the numerical modeling of crystalline rock salt remains an active research area. Analysis of complex multiphysical responses of the natural and reconsolidated salt is an integral component of the design of short- and long-term life cycle of a salt repository. In the repository, the facility may have various phases of operation cycles that involve plugging, sealing, testing, and reconsolidation of the saline materials [6]. Under such conditions, the reconsolidated salt is influenced by moisture content, brine inclusions inside halite crystals, and other materials, such as clay or impurity, that might present in the grain boundary. Within certain temperature and confining pressure ranges (between 250 and $790{ }^{\circ} \mathrm{C}$ and between 0.15 and $12 \mathrm{MPa}$ ), the responses of salt may become even more complicated due to re-crystallization and grain boundary migration. This generally leads to precipitation creeping [12]. A number of previous studies, therefore, investigate the phenomenology and micro-mechanisms associated with these mechanical behaviors of salt (e.g., Carter et al. [13], Carter and Hansen [14], Urai et al. [15], Carter et al. [16], Senseny et al. [17], Urai et al. [18], Hansen et al. [19]). These studies have explored the dislocation, dissolution-precipitation creep, healing behavior, and flow transport properties of natural or reconsolidated salt under a wide range of load and temperature conditions in a phenomenological setting where microstructural attributes of the rock salt are not explicitly modeled.

Since salt formations found in nature often contain varying amounts of impurities, minerals, and brine inclusion, how to capture these spatial heterogeneous effects without explicitly modeling its microstructure remains a major challenge. Various phenomenological constitutive models have been proposed to capture the macroscopic responses of salt (e.g., Munson and Dawson [20], Senseny et al. [17], Chan et al. [21], Miao et al. [22], Munson [23], Chan et al. [5], Aubertin et al. [24], Olivella and Gens [25], Zhu and Arson [26], Broome et al. [27], Shen et al. [28]). 
For instance, Olivella et al. [29] propose a general formulation for nonisothermal multiphase flow of gas and brine in saline media, which was further extended into a series of works for coupled thermo-hydro-mechanical analysis of saline materials (e.g., Olivella et al. [30,31], Olivella and Gens [32]). Although they did not explicitly incorporate the grain boundary, damage and healing, and the microstructural information, the complicated history- and rate-dependent behaviors of saline media as well as the multiphase interactions among constituents including gas, liquid, and solid were addressed by linking fluid flow, permeability change, thermal gradient, and phenomenological constitutive law for the solid skeleton.

While these phenomenological approaches inspired by micromechanics of crystals have achieved a level of success in the past, a more physically consistent forward prediction may require stronger physical underpinnings such that the interactions among the impurity, the precipitated brine and the anisotropy of crystalline constitutive responses can be captured properly [6]. Yet, bridging the multiscale coupling effects from the grain scale to the field applications remains a challenging task due to the complexity of the physical nature of rock salt. Our ultimate goal is to create a multiscale polycrystalline material model suitable for field-scale applications. As a first step toward this goal, this article will focus on modeling the thermo-mechanical response of single-crystal halite. In this paper, our objective is to derive, implement, and validate an elastoplastic model for single-crystal halite subjected to different thermal and mechanical loadings relevant to nuclear waste disposal applications. We will incorporate other important multiphysics and multiscale polycrystalline mechanisms such as the interactions across grain boundaries, the intra-crystalline and inter-crystalline brine inclusion, and the role of the impurity and additive on the precipitating and pressure solution of the grain boundaries in future contributions of this series of works.

We present a unified mathematical framework that enables us to capture the anisotropic inelastic brittle and ductile behaviors of single-crystal halite. We achieve this by combining (1) a thermal-sensitive rate-dependent crystal plasticity formulation that captures the anisotropic plastic deformation caused by the slip of crystallographic planes and (2) a multi-phase-field regularized fracture model that captures interactions of the anisotropic intragranular and transgranular fractures of single-crystal halite under the temperature range relevant to nuclear waste disposal applications (e.g., the WIPP). The intrinsic anisotropy of halite stemming from the microstructure is incorporated into the crystal plasticity theory. This theory is a micromechanics-based constitutive law where the anisotropic plastic flow is associated with activation(s) of the slip systems oriented according to the lattice structure of the crystalline materials [33-37]. In this work, we use the strain energy equivalence theory such that the crystal plasticity framework can be integrated into a phase field model capturing the evolution of damage. Because the creeping mechanisms, such as grain boundary diffusion, dislocation creep, and thermal activated glide, are highly sensitive to temperature, the temperature-dependent energy dissipation due to creeping is incorporated into the crystal plasticity model (cf. Cuitino and Ortiz [38], Borja and Wren [39], Anand and Kothari [40], Miehe and Schröder [41], Borja and Rahmani [42], Tjioe and Borja [43]). The damage and crack growth of single-crystal materials are captured via the phase field approach, which is a sub-class of the smeared crack approach. This method allows us to capture complicated crack patterns without introducing an embedded discontinuity (e.g., Miehe et al. [44,45], Clayton and Knap [46,47], Na et al. [48]). It should be noted that incorporation of the phase field model is not the only feasible way to model cleavage fractures. Other methods, such as the introduction of pseudo-slip in crystalline materials, (e.g., Aslan et al. [49], Clayton [50]) have been used to model the coupled effect of single crystal plasticity and damage.

We use a sub-class of the phase field model that employs multiple phase fields to capture damage accumulated in different directions. The multi-phase-field approach has been very popular to model a wide spectrum of material behaviors including anisotropic responses of regularized interfaces for planar dislocation (e.g., Koslowski and Ortiz [51]), re-crystallization (e.g. Takaki et al. [52]), and anisotropic fracture (e.g., Oshima et al. [53], Nguyen et al. [54,55]). See also Steinbach [56] for a comprehensive review. In the content of anisotropic phase field models, one obvious advantage of the multi-phase-field approach is the lack of higher-order terms that are typically required for the single-phase-field counterpart to capture non-convex anisotropic fracture energies [57,58]. As a result, the multi-phase-field model does not require specific finite element spaces spanned by piece-wise smooth and globally $C^{1}$-continuous basis functions. Instead, a simple low-order finite element can be used. This simplicity, although offset by the additional computational cost due to the additional degrees of freedom, motivates us to use the multi-phase-field model in this work. The number of independent phase fields and their directional features are assumed to be consistent with the slip planes of halite. Finally, the crystal plasticity theory and the multi-phase-field approach are incorporated into a set of multi-physical field equations. The formulations include the balance of linear momentum, the micro-force equations for plastic slip and multiple phase-fields, and the balance of energy. An operator-split integration scheme is introduced to construct a feasible algorithm for numerical simulations. 
The organization of the paper is as follows. In Section 2, we first describe the kinematics of a halite grain with multiple slip systems. Then, the application of effective stress equivalence theory on the multi-phase-field model is discussed with respect to anisotropic fracture for crystalline materials. Within this mathematical framework, the general governing equations including the balance of linear momentum, microforce equations, and balance of energy are derived. The specific choice of a free energy function that combines the crystal plasticity and the multi-phase-field approaches is provided. Subsequently, in Section 3, the stress update algorithm in effective stress space is described followed by the description of both rate-dependent and rate-independent settings. In Section 4, the finite element formulation, as well as solution strategies for nonlinear systems of equations, are presented. Numerical examples are then provided in Section 6, followed by a conclusion.

As for notations and symbols, bold-faced letters denote tensors; the symbol '.' denotes a single contraction of adjacent indices of two tensors (e.g., $\boldsymbol{a} \cdot \boldsymbol{b}=a_{i} b_{i}$ or $\boldsymbol{c} \cdot \boldsymbol{d}=c_{i j} d_{j k}$ ); the symbol ' $:$ ' denotes a double contraction of adjacent indices of tensor of rank two or higher (e.g., $\left.\boldsymbol{C}: \boldsymbol{\epsilon}^{e}=C_{i j k l} \epsilon_{k l}^{e}\right)$; the symbol ' $\otimes$ ' denotes a juxtaposition of two vectors (e.g., $\boldsymbol{a} \otimes \boldsymbol{b}=a_{i} b_{j}$ ) or two symmetric second order tensors (e.g., $\left.(\boldsymbol{\alpha} \otimes \boldsymbol{\beta})=\alpha_{i j} \beta_{k l}\right)$. As for sign conventions, unless specify otherwise, we consider the direction of the tensile stress and dilative pressure as positive.

\section{Governing equations}

In this section, we present the field equations that capture the thermo-mechanical coupling effect of single-crystal halite. We begin by reviewing kinematics of deformation of a crystalline solid with multiple slip systems. To capture the brittle-ductile transition and the brittle fractures that might occur under low confining pressure, a multi-phase-field model is used. Adopting the ideas originated from Nguyen et al. [55], we introduce multiple phase fields to model anisotropic damage such that each phase field represents the damage along a particular preferential direction. Finally, the coupled anisotropic damage-plasticity behavior of single-crystal halite is captured via the effective stress $[59,60]$. The use of effective stress concept in the coupled phase field and plasticity modeling of geological materials can be also found in Choo and Sun [61]. The total stress in a damaged configuration is then recovered based on the effective stress and the anisotropic gradient damage computed from the phase fields. Meanwhile, the field theory for balances of linear momentum microforce and energy is discussed.

\subsection{Strain energy equivalence for coupling phase field and plasticity}

In this work, an anisotropic phase field fracture framework is coupled with a crystal plasticity model to simulate the anisotropic path-dependent behavior of single-crystal halite. Here we adopt the strain energy equivalence principle used previously in isotropic and anisotropic damage mechanics $[60,62,63]$. This principle hypothesizes that there exists a fictitious undamaged counterpart of the damaged halite such that a body composed of the fictitious undamaged material will experience an effective stress $\hat{\sigma}$ while the actual body will experience a stress $\sigma$. Furthermore, we assume that despite the damage characterized by the multiple phase fields is anisotropic, the effective stress and the total stress are co-axial. Consequentially, the degradation function $g(\underline{d})$ becomes a scalar function of the collection of phase fields $\underline{d}$. The relationship between the total and effective stresses when both the fictitious and the real material exhibit the same infinitesimal strain (i.e., $\boldsymbol{\epsilon}=\hat{\boldsymbol{\epsilon}}$ ) is,

$$
\sigma=g(\underline{d}) \hat{\sigma},
$$

and the corresponding infinitesimal stress rate is,

$$
\dot{\boldsymbol{\sigma}}=\sum_{i=1}^{N} \frac{\partial g(\underline{d})}{\partial d_{i}} \dot{d}_{i} \hat{\boldsymbol{\sigma}}+g(\underline{d}) \dot{\hat{\boldsymbol{\sigma}}},
$$

where $d_{i},(i=1,2, \ldots, N)$, is the phase field for each preferential direction and $N$ is the total number of phase fields. An important implication of (1) is that while damage and plastic processes remain coupled, the plasticity model can be associated with the fictitious undamaged body. Hence one may use a decoupled plasticity algorithm to first obtain the effective stress from the strain field then update the total stress using the degradation function from each material point. 

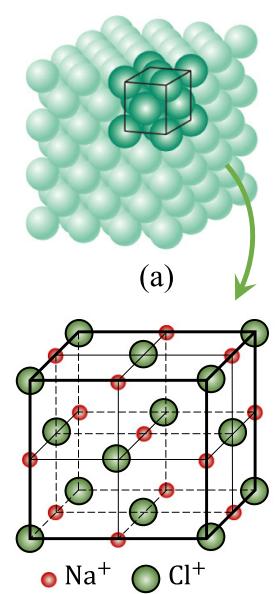

(b)

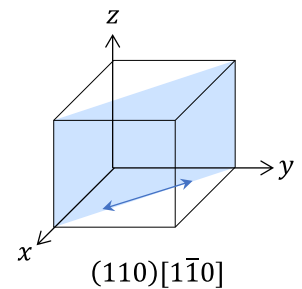

(110) $[1 \overline{1} 0]$

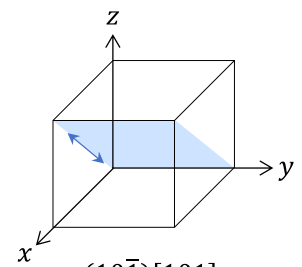

$(10 \overline{1})[101]$

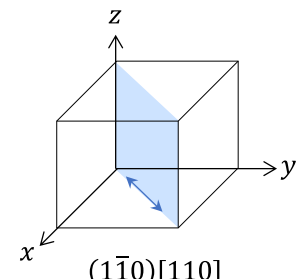

(11̄0)[110]

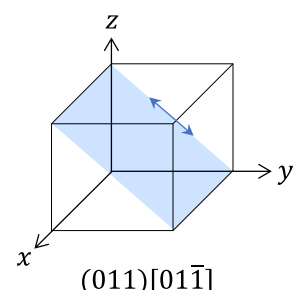

(c)
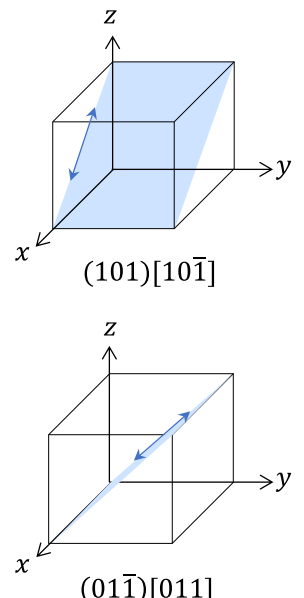

(011) $[011]$

Fig. 2. The description of the slip-system of single-crystal halite, (a) an aggregate of many atoms for the face centered cubic crystal structure (reproduced from Callister Jr. and Rethwisch [68] — adapted from the original Moffat et al. [69], (b) a unit cell for the sodium chloride (NaCl) crystal structure (reproduced from Callister Jr. and Rethwisch [68]), and (c) the slip-system $\{110\}\langle 1 \overline{1} 0\rangle$.

\subsection{Kinematics for crystal deformation of the damaged halite}

For completeness, we briefly review the kinematics of crystal deformation and restrict the formulation within the infinitesimal range. More comprehensive treatment of this subject can be found in, for instance, Budiansky and Wu [64], Borja and Wren [39], Miehe and Schröder [41], Borja [65]. Recall that we adopt the approach in Simo and Ju [63] in which we assume that the actual (damaged) crystal and the fictitious counterpart undertake the same strain and strain history.

The elastoplastic deformation of a single-crystal grain is attributed to the interactions between crystal blocks along the predefined crystallographic planes in the slip system. A slip-system is defined by a combination of crystallographic planes (i.e., slip planes) and the corresponding sliding directions (i.e., slip directions). The atomic arrangement of the slip system of crystalline materials leads to a preferential direction of the plastic flow, hence the overall constitutive response of a single crystal is anisotropic. In Face Cubic Centered (FCC) crystals, for example, the slip-systems are defined by the densest packing planes in terms of atomic arrangement as the slip-plane $\{111\}$ and slip-direction $\langle 110\rangle$. This dense packing causes FCC materials, such as aluminum, copper, gold, and silver to exhibit higher ductility than the Body Cubic Center (BCC) crystals such as, iron and chromium (although packing alone is not the only factor that influences ductility) [65]. One may view the halite structure as a FCC with secondary atoms in its octahedral holes (cf. Williams [66]). As a result, a set of the slip-planes is known to be $\{110\}$ (rather than $\{111\}$ for FCC), and the corresponding slip directions are $\langle 1 \overline{1} 0\rangle$. Please see the slip-system of halite in Fig. 2. This set of planes is electrically neutral, and the motion on these planes avoids charged layers gliding over one another (cf. Hansen et al. [19], Carter and Norton [67]).

We consider a continuum body $(\mathcal{B})$ with material points identified by the position vectors $x \in \mathcal{B}$. The displacement of a material point with time $t$ can be denoted by $\boldsymbol{u}(\boldsymbol{x}, t)$, and we define the strain measure $(\boldsymbol{\epsilon})$ as the symmetric part of the displacement gradient, i.e.,

$$
\boldsymbol{\epsilon}:=\nabla^{\mathrm{sym}} \boldsymbol{u}=\frac{1}{2}\left(\nabla \boldsymbol{u}+(\nabla \boldsymbol{u})^{\mathrm{T}}\right) .
$$

The additive decomposition of the infinitesimal total strain leads to,

$$
\boldsymbol{\epsilon}=\boldsymbol{\epsilon}^{e}+\boldsymbol{\epsilon}^{p}+\boldsymbol{\epsilon}^{\theta}
$$

where $\epsilon^{e}$ and $\epsilon^{p}$ indicate the elastic and plastic components, respectively.

Meanwhile, the thermal component is assumed to be isotropic and defined as a function of the thermal expansion coefficient, i.e., $\boldsymbol{\epsilon}^{\theta}=\alpha\left(\theta-\theta_{o}\right) \boldsymbol{I}$, where $\alpha$ is the thermal expansion coefficient; $\theta_{o}$ is a reference temperature at which 
the thermal strain $\boldsymbol{\epsilon}^{\theta}=\mathbf{0}$ (cf. Aldakheel [70], Clayton [50], Miehe et al. [71]). In crystalline materials, the elastic strain describes distortion of the lattice structure which vanishes when the applied stress is released. On the other hand, the plastic strain is irreversible and evolves due to the slip on crystallographic planes. In this work, we assume the thermal expansion as an elastic response and consider it in the elastic part of free energy functional in (41) followed by Anand and Gurtin [72].

The $\alpha$-slip system is composed of (1) $\boldsymbol{m}^{\alpha}$, the unit normal to a crystallographic plane and (2) $\boldsymbol{s}^{\alpha}$, the direction of plastic slip on that plane. Therefore, the slip-system $\alpha$ is defined by orthonormal vectors $\left(\boldsymbol{m}^{\alpha}, \boldsymbol{s}^{\alpha}\right)$ based on the crystallographic features of crystals. We then obtain the plastic strain by summing over all crystallographic slips,

$$
\boldsymbol{\epsilon}^{p}=\sum_{\alpha} \gamma^{\alpha} \boldsymbol{S}^{\alpha}
$$

where $\gamma^{\alpha}$ indicates the plastic slip corresponding to the slip-system $\alpha . S^{\alpha}$ denotes the symmetric part of the Schmid tensor (i.e., $\boldsymbol{m}^{\alpha} \otimes \boldsymbol{s}^{\alpha}$ ), which can be expressed as follows,

$$
\boldsymbol{S}^{\alpha}=\frac{1}{2}\left(\boldsymbol{m}^{\alpha} \otimes \boldsymbol{s}^{\alpha}+\boldsymbol{s}^{\alpha} \otimes \boldsymbol{m}^{\alpha}\right) .
$$

Note that the summation convention is not employed in respect of indices relating to the slip systems. Furthermore, the thermal expansion and structural heating are included by using a thermal-sensitive elastic stored energy function for thermoelastic coupling in (40). For crystals with cubic symmetry crystals, the second-order material tensors such as thermal expansion and conductivity are all symmetric with one independent component (cf. Clayton [73]). For other types of crystals, the anisotropy of any symmetric second-order material tensors can be introduced via spectral decomposition. In this case, one may assume that the principal crystallographic directions are parallel to the eigenvectors of the material tensors (cf. Meissonnier et al. [74]). The thermal effect on the inelastic behavior of single-crystal halite is incorporated via the temperature-dependent flow rule for the plastic slips (Section 2).

\subsection{Multi-phase-field approximation for anisotropic fracture}

The phase field fracture modeling can be considered as a regularized smeared approach. In this approach the cracks are not explicitly captured via embedded discontinuities but approximated by an implicit indicator function obtained from regularizing the strong discontinuities with a characteristic length [75-77]. Here we assume that this characteristic length is sufficiently smaller than the grain size but larger than the finite element mesh size used for numerical simulations. In those cases, the strongly anisotropic damage and fracture behaviors can be captured either via a single phase field within a higher-order Cahn-Hilliard framework (e.g., Clayton and Knap [47], Li et al. [57], Teichtmeister et al. [58]) or via multiple phase fields, each constrained by governing equation without the fourth-order terms (e.g., Nguyen et al. [54,55]). Due to the simplicity of the latter approach, we adopt it to capture the damage and fracture behavior in single-crystal halite.

In a regularized framework where the sharp crack topology is approximated by a diffusive representation, the phasefield variable $(d(\boldsymbol{x}, t) \in[0,1])$ is an implicit function whose value indicates the location of the smeared $\operatorname{crack}(\mathrm{s})$. Let $\mathcal{T}$ denote a set of discontinuous fractures inside a body $\mathcal{B}$. Then the total area of the crack surfaces can be described by an area integral over $\mathcal{T}$. By introducing a crack density function $\Gamma_{l}(d, \nabla d)$ the total crack area can be described by the volume integral as,

$$
\Gamma=\int_{\mathcal{T}} d A \approx \int_{\mathcal{B}} \Gamma_{l}(d, \nabla d) d V \text { where } \Gamma_{l}(d, \nabla d):=\frac{1}{2 l} d^{2}+\frac{l}{2}|\nabla d|^{2} .
$$

Here $l$ indicates a length scale that controls the width of the smooth approximation of the crack. The assigned scalar phase-field values 0 and 1 represent the intact and completely damaged region, respectively. The phase-field value between $0<d<1$ indicates a partially damaged material state at the corresponding material point. The regularized crack surface can be further extended to the anisotropic case as,

$$
\Gamma_{l}(d, \nabla d, w):=\frac{1}{2 l} d^{2}+\frac{l}{2} \omega:(\nabla d \otimes \nabla d),
$$


where $\omega$ is a second-order structural tensor, which is invariant with respect to rotations for characterizing the material anisotropy [47]. This tensor can be defined as,

$$
\omega=\mathbf{1}+\beta(\mathbf{1}-\boldsymbol{m} \otimes \boldsymbol{m}),
$$

where 1 denotes the second-order identity tensor; $m$ indicates the unit normal vector to the potentially preferred cleavage plane; $\beta \gg 1$ is the parameter penalizing fracture on planes not normal to the unit vector $\boldsymbol{m}$. The isotropic crack surface energy function in (7) can be recovered by setting $\beta=0$.

To capture the anisotropy inherent in the crystalline materials, we associate the orientations of each slip plane as a potential cleavage plane. Adopting the multi-phase-field technique firstly introduced by Oshima et al. [53] and further developed in Nguyen et al. [54,55], we assign multiple phase fields $\underline{d}=\left\{d_{1}, d_{2}, \ldots, d_{n}\right\}$ to quantify the damage accumulation on each slip plane normal to the unit vector $\boldsymbol{m}^{i}$. Therefore, the total crack length can be defined by the summation of each anisotropic crack density function which is rewritten as [54,55],

$$
\begin{gathered}
\Gamma_{l}(\underline{d}, \nabla \underline{d}, \underline{\omega})=\sum_{i}\left[\frac{1}{2 l}\left(d_{i}\right)^{2}+\frac{l}{2} \boldsymbol{\omega}_{i}:\left(\nabla d_{i} \otimes \nabla d_{i}\right)\right], \\
\text { where } \boldsymbol{\omega}_{i}=\mathbf{1}+\beta\left(\mathbf{1}-\boldsymbol{m}^{i} \otimes \boldsymbol{m}^{i}\right) .
\end{gathered}
$$

For convenience, we use the underline to denote a set of variables. In analogy to (6), $\boldsymbol{m}^{i}$ corresponds to $\boldsymbol{m}^{\alpha}-$ the unit normal vector of each crystallographic slip plane. The length scale $l$ and penalizing parameter $\beta$ are assumed to be the same for each slip system. These parameters may further differentiate for each slip system based on the microscopic information of crystalline materials.

\subsection{Balance of linear momentum and microforce}

In this section, we derive the balance of linear momentum and microforce for single crystals with damage via the principle of virtual power. The virtual-power formulation of the single crystal using microforce balance is well established and has been described in the literature (cf. Gurtin et al. [36]). Based on this standard theory, De Lorenzis et al. [78] extended the virtual power formulation by introducing an additional microforce balance associated with a crack phase field. Our new contribution is to extend this work for the multi-phase-field formulation coupled with crystal plasticity. We postulate the existence of multiple microforces, each microforce conjugates to a phase field for anisotropic damage in a single crystal. Furthermore, we extend this framework to incorporate thermal diffusion such that non-isothermal condition can be simulated. For simplicity, we limit our analysis within the small deformation range and the small temperature difference assumption (cf. Anand and Gurtin [72]). As described in Section 2.2, the thermal expansion effect is included in the elastic stored energy function (41) based on the standard virtual power formulation [72]. This treatment may be sufficient for a geological disposal under the limited temperature change condition for low-level radioactive waste materials, for example, generated by military activities (e.g., the Waste Isolation Pilot Plant, WIPP) [6,79].

The standard derivation procedure using the principle of virtual power for phase-field modeling of fracture in a single-crystal is described in great detail in Gurtin et al. [36], De Lorenzis et al. [78]. Here we briefly outline the expression of virtual power formulations including macroforce (i.e., the total Cauchy stress), microforce for a single crystal, and additional microforces for multiple phase fields associated with the resultant balance equations. In a domain $\mathcal{B}$, the internal power $(\mathcal{I})$ over a subregion $\mathcal{P} \subset \mathcal{B}$ may take the form of,

$$
\mathcal{I}(\mathcal{P})=\int_{\mathcal{P}} \sigma: \dot{\boldsymbol{\epsilon}}^{e} d V+\sum_{\alpha} \int_{\mathcal{P}} \pi^{\alpha} \dot{\gamma}^{\alpha} d V+\sum_{i}\left(\int_{\mathcal{P}} \xi_{i} \cdot \nabla \dot{d}_{i} d V+\int_{\mathcal{P}} \pi_{i} \dot{d}_{i} d V\right)
$$

where $\sigma$ indicates the Cauchy stress with the power-conjugate to $\dot{\boldsymbol{\epsilon}}^{e} ; \pi^{\alpha}$ denotes the microscopic force associated with the slip rate $\dot{\gamma}^{\alpha} ; \xi_{i}$ is the microscopic stress power-conjugate to $\nabla \dot{d}_{i}$; and $\pi_{i}$ is the microscopic internal body force (power conjugate to $\dot{d}_{i}$ ). Note that the summation convention is not employed regarding indices related to multiple phase-fields. Each phase-field variable corresponds to the direction of a crystalline slip system. As mentioned in Section 2.1, $\sigma$ is a total stress considering damage to the material obtained by (1). The calculation of the total stress and a stress update algorithm will be explained in a later section. Now we assume that the external power $(\mathcal{W})$ acting 
upon $\mathcal{P} \subset \mathcal{B}$ has the form as,

$$
\mathcal{W}(\mathcal{P})=\int_{\partial \mathcal{P}} \boldsymbol{t}(\boldsymbol{n}) \cdot \dot{\boldsymbol{u}} d A+\int_{\mathcal{P}} \boldsymbol{b} \cdot \dot{\boldsymbol{u}} d V+\sum_{i}\left(\int_{\partial \mathcal{P}} \chi_{i}(\boldsymbol{n}) \cdot \dot{d}_{i} d A+\int_{\mathcal{P}} \lambda_{i} \dot{d}_{i} d A\right),
$$

where $\boldsymbol{t}$ is the traction vector with outward unit normal $\boldsymbol{n} ; \boldsymbol{b}$ is the macroscopic body force; $\chi_{i}$ and $\lambda_{i}$ are the microscopic external traction and body force, respectively, both power-conjugate to $\dot{d}_{i}$. Note that a scalar external virtual microscopic force power conjugate to $\dot{\gamma}^{\alpha}$ is not considered [36]. An additional assumption is made such that each field, that is $\dot{\boldsymbol{u}}, \dot{\boldsymbol{\epsilon}}^{e}, \dot{\gamma}^{\alpha}$, and $\dot{d}_{i}$, is known at some arbitrary known but at fixed time and can be independently specified within the kinematic constraints, (3) to (5), as

$$
\nabla^{\mathrm{sym}} \tilde{\boldsymbol{u}}=\tilde{\boldsymbol{\epsilon}}^{e}+\sum_{\alpha} \tilde{\gamma}^{\alpha} \boldsymbol{S}^{\alpha}
$$

By denoting each virtual field by $\tilde{\boldsymbol{u}}, \tilde{\boldsymbol{\epsilon}}^{e}, \tilde{\gamma}^{\alpha}$, and $\tilde{d}_{i}$, a generalized virtual velocity $\mathcal{V}$ can be defined by $[36,72,78]$,

$$
\mathcal{V}=\left(\tilde{\boldsymbol{u}}, \tilde{\boldsymbol{\epsilon}}^{e}, \underline{\tilde{\gamma}}, \underline{\tilde{d}}\right) \text {. }
$$

Then the principle of virtual power is the requirement that, $\mathcal{I}(\mathcal{P}, \mathcal{V})=\mathcal{W}(\mathcal{P}, \mathcal{V})$, that is,

$$
\begin{aligned}
& \int_{\mathcal{P}} \boldsymbol{\sigma}: \tilde{\boldsymbol{\epsilon}}^{e} d V+\sum_{\alpha} \int_{\mathcal{P}} \pi^{\alpha} \tilde{\gamma}^{\alpha} d V+\sum_{i}\left(\int_{\mathcal{P}} \boldsymbol{\xi}_{i} \cdot \nabla \tilde{d}_{i} d V+\int_{\mathcal{P}} \pi_{i} \tilde{d}_{i} d V\right) \\
& =\int_{\partial \mathcal{P}} \boldsymbol{t}(\boldsymbol{n}) \cdot \tilde{\boldsymbol{u}} d A+\int_{\mathcal{P}} \boldsymbol{b} \cdot \tilde{\boldsymbol{u}} d V+\sum_{i}\left(\int_{\partial \mathcal{P}} \chi_{i}(\boldsymbol{n}) \cdot \tilde{d}_{i} d A+\int_{\mathcal{P}} \lambda_{i} \tilde{d}_{i} d A\right),
\end{aligned}
$$

for all virtual velocities $\mathcal{V}$. Again, the derivation of balance equations is straightforward (e.g., Gurtin et al. [36], De Lorenzis et al. [78], Anand and Gurtin [72]), thus resulting in the following final expressions:

$$
\begin{aligned}
& \nabla \cdot \boldsymbol{\sigma}+\boldsymbol{b}=\mathbf{0}, \quad \text { (Linear momentum) } \\
& \boldsymbol{t}(\boldsymbol{n})=\boldsymbol{\sigma} \cdot \boldsymbol{n}, \quad \text { (Macroscopic traction) } \\
& \pi^{\alpha}=\tau^{\alpha} \text { with } \tau^{\alpha}=\boldsymbol{S}^{\alpha}: \boldsymbol{\sigma}, \quad \text { (Microscopic force balance for each slip system) } \\
& \nabla \cdot \boldsymbol{\xi}_{i}-\pi_{i}+\lambda_{i}=0, \quad \text { (Microscopic force balance for each phase field) } \\
& \chi_{i}(\boldsymbol{n})=\boldsymbol{\xi}_{i} \cdot \boldsymbol{n} . \quad \text { (Phase-field microscopic traction) }
\end{aligned}
$$

Here $\tau^{\alpha}$ indicates the Schmid stress or the resolved shear stress, which represents the macroscopic stress $\sigma$ resolved on the slip system $\alpha$. In addition, the number of (19) and (20) is consistent with the number of each phase-field corresponding to the slip system of single-crystal halite. We further note that the macroforces and microforces are related based on (18) such that $\tau^{\alpha}$ represents the force applied on the lattice of a single crystal for dislocations on the slip system $\alpha$ while $\pi^{\alpha}$ constitutes internal forces on the slip system $\alpha$ [36]. The multiple phase-field variables, however, do not show clear relations associated with the macroforce and the microforce on the slip systems. This interaction is established based on our choice of a free energy functional, which will be covered in a later section.

\subsection{Energy balance equation and dissipation inequality}

To capture the thermo-mechanical behavior of a single crystal, we present an energy balance equation which includes contributions of heat conduction, mechanical dissipation, and structural heating. The first law (energy balance) can be expressed in a local form,

$$
\dot{e}=\boldsymbol{\sigma}: \dot{\boldsymbol{\epsilon}}-\nabla \cdot \boldsymbol{q}+r_{\theta},
$$

where $e$ is internal energy per unit volume; $\boldsymbol{q}$ is the heat flux vector; $r_{\theta}$ is the heat source term. This equation can be rewritten in greater detail by including the dissipation of the plastic slip and the work done by the growth of phase field (15). Recall that the rate of the phase-field change is power conjugate to the microforce (cf. Gurtin [80], Borden [81]). The balance of energy, therefore, reads,

$$
\dot{e}=\sigma: \dot{\boldsymbol{\epsilon}}^{e}+\sum_{\alpha} \pi^{\alpha} \dot{\gamma}^{\alpha}+\sum_{i}\left(\xi_{i} \cdot \nabla \dot{d}_{i}+\pi_{i} \dot{d}_{i}\right)-\nabla \cdot \boldsymbol{q}+r_{\theta}
$$


Then the second law of thermodynamics (Clausius-Duhem inequality) is,

$$
\mathscr{D}_{\text {int }}=\dot{\eta}+\nabla \cdot\left(\frac{\boldsymbol{q}}{\theta}\right)-\frac{r_{\theta}}{\theta} \geq 0
$$

in which $\eta$ is an entropy term, and $\theta$ is absolute temperature. Here the flux vector $(\boldsymbol{q})$ can be expressed using Fourier's law for heat conduction,

$$
\boldsymbol{q}=-\boldsymbol{\kappa} \cdot \nabla \theta,
$$

where $\boldsymbol{\kappa}$ is the thermal conductivity tensor. In addition, we may recall the Helmholtz free energy $(\psi)$ which shows the relation between the internal energy $(e)$ and entropy $(\eta)$ as,

$$
\psi=e-\theta \eta .
$$

Therefore, the dissipation inequality $\left(\mathscr{D}_{\text {int }}\right)$ can be rewritten as,

$$
\mathscr{D}_{\text {int }}=\sigma: \dot{\boldsymbol{\epsilon}}^{e}+\sum_{\alpha} \pi^{\alpha} \dot{\gamma}^{\alpha}+\sum_{i}\left(\xi_{i} \cdot \nabla \dot{d}_{i}+\pi_{i} \dot{d}_{i}\right)-(\eta \dot{\theta}+\dot{\psi})-\frac{1}{\theta} \boldsymbol{q} \cdot \nabla \theta \geq 0 .
$$

Now we consider the Helmholtz free energy function for thermo-elasto-plastic crystal materials of the following form,

$$
\psi=\hat{\psi}\left(\boldsymbol{\epsilon}^{e}, \underline{s}, \theta, \underline{d}, \nabla \underline{d}\right),
$$

where $\boldsymbol{\epsilon}^{e}$ is the elastic strain; $\theta$ the temperature; $\underline{d}$ a set of crack phase-fields and its gradient $\nabla \underline{d}$; $\underline{s}$ a set of a local scalar measure related to the plastic slip accumulation for each slip system defined by,

$$
\dot{s}^{\alpha}:=\dot{\gamma}^{\alpha} \geq 0 \text { where } s^{\alpha}(t)=\int_{0}^{t} \dot{\gamma}^{\alpha} d \tau .
$$

The substitution of (27) into (26) induces the dissipation inequality as the following form,

$$
\begin{aligned}
& \left(\boldsymbol{\sigma}-\frac{\partial \hat{\psi}}{\partial \boldsymbol{\epsilon}^{e}}\right): \dot{\boldsymbol{\epsilon}}^{e}+\sum_{\alpha} \pi^{\alpha} \dot{\gamma}^{\alpha}-\sum_{\alpha} \frac{\partial \hat{\psi}}{\partial s^{\alpha}} \dot{s}^{\alpha}+\sum_{i}\left(\pi_{i}-\frac{\partial \hat{\psi}}{\partial d_{i}}\right) \dot{d}_{i} \\
& +\sum_{i}\left(\boldsymbol{\xi}_{i}-\frac{\partial \hat{\psi}}{\partial \nabla d_{i}}\right) \cdot \nabla \dot{d}_{i}-\left(\eta+\frac{\partial \hat{\psi}}{\partial \theta}\right) \dot{\theta}-\frac{1}{\theta} \boldsymbol{q} \cdot \nabla \theta \geq 0 .
\end{aligned}
$$

Well-known arguments (e.g., Standard Coleman-Noll arguments) lead to the constitutive relations for the Cauchy stress and entropy, respectively,

$$
\boldsymbol{\sigma}=\frac{\partial \hat{\psi}}{\partial \boldsymbol{\epsilon}^{e}}, \eta=-\frac{\partial \hat{\psi}}{\partial \theta} .
$$

We then further derive the dissipation inequality by following the individual group terms. The inequality related to each phase-field variable can be expressed as,

$$
\sum_{i}\left(\pi_{i}-\frac{\partial \hat{\psi}}{\partial d}\right) \dot{d}_{i}+\sum_{i}\left(\xi_{i}-\frac{\partial \hat{\psi}}{\partial \nabla d_{i}}\right) \cdot \nabla \dot{d}_{i} \geq 0,
$$

which leads to the phase-field microscopic constitutive equations as,

$$
\pi_{i}=\frac{\partial \hat{\psi}}{\partial d_{i}} \text { and } \xi_{i}=\frac{\partial \hat{\psi}}{\partial \nabla d_{i}}
$$

Substitution of (32) into (19) with the assumptions of no microscopic body force $\left(\lambda_{i}=0\right)$ leads to the multi-phase-field equations as,

$$
\nabla \cdot\left(\frac{\partial \hat{\psi}}{\partial \nabla d_{i}}\right)-\frac{\partial \hat{\psi}}{\partial d_{i}}=0 .
$$

Note that previous phase field fracture models, such as the time-regularized viscous crack propagation mode in Miehe et al. [44], often introduce dissipation function that depends on rate of phase field and that of the phase field 
gradient. Such an extension is out of the scope of this study but will be considered in the future. Interested readers are referred to previous works, such as Miehe et al. [44], Mota et al. [82], Stainier and Ortiz [83], Yang et al. [84], for a variational consistent way to introduce rate-dependence for path-dependent materials. Based on (30) and (32), the reduced dissipation inequality becomes,

$$
\underbrace{\sum_{\alpha} \pi^{\alpha} \dot{\gamma}^{\alpha}-\sum_{\alpha} \frac{\partial \hat{\psi}}{\partial s^{\alpha}} \dot{s}^{\alpha}}_{D_{p}}-\frac{1}{\theta} \boldsymbol{q} \cdot \nabla \theta \geq 0
$$

where $D_{p}$ denotes the plastic dissipation rate. Considering the definition of $g^{\alpha}$, the inequality (34) can be further reduced to,

$$
\sum_{\alpha}\left(\pi^{\alpha} \dot{\gamma}^{\alpha}+g^{\alpha} \dot{s}^{\alpha}\right)-\frac{1}{\theta} \boldsymbol{q} \cdot \nabla \theta \geq 0, \quad \text { where } g^{\alpha}=-\frac{\partial \hat{\psi}}{\partial s^{\alpha}},
$$

in which the thermodynamic force $g^{\alpha}$ power-conjugate to $\dot{s}^{\alpha}$ is introduced. Finally, the balance of energy is obtained by substituting the free energy (25) into (22):

$$
\dot{\psi}+\dot{\eta} \theta+\eta \dot{\theta}-\boldsymbol{\sigma}: \dot{\boldsymbol{\epsilon}}^{e}-\sum_{\alpha} \pi^{\alpha} \dot{\gamma}^{\alpha}-\sum_{i}\left(\xi_{i} \cdot \nabla \dot{d}_{i}+\pi_{i} \dot{d}_{i}\right)+\nabla \cdot \boldsymbol{q}-r_{\theta}=0 .
$$

This equation can be rewritten by combining the constitutive relations of (30) and (32) as,

$$
\begin{aligned}
-\theta \frac{\partial^{2} \hat{\psi}}{\partial \theta^{2}} \dot{\theta}= & \sum_{\alpha}\left(\pi^{\alpha} \dot{\gamma}^{\alpha}+g^{\alpha} \dot{s}^{\alpha}\right)+\sum_{\alpha}\left(\theta \frac{\partial^{2} \hat{\psi}}{\partial \theta \partial s^{\alpha}} \dot{s}^{\alpha}\right)+\sum_{i}\left(\frac{\partial^{2} \hat{\psi}}{\partial \theta \partial d_{i}} \dot{d}_{i}+\frac{\partial^{2} \hat{\psi}}{\partial \theta \partial \nabla d_{i}} \cdot \nabla \dot{d}_{i}\right) \theta \\
& +\theta\left(\frac{\partial^{2} \hat{\psi}}{\partial \theta \partial \boldsymbol{\epsilon}^{e}}: \dot{\boldsymbol{\epsilon}}^{e}\right)-\nabla \cdot \boldsymbol{q}+r_{\theta} .
\end{aligned}
$$

Considering a choice of a free energy function in (40), we may reduce the equation as,

$$
\underbrace{-\theta \frac{\partial^{2} \hat{\psi}}{\partial \theta^{2}}}_{c_{v}} \dot{\theta}=\underbrace{\sum_{\alpha}\left(\pi^{\alpha} \dot{\gamma}^{\alpha}+g^{\alpha} \dot{\boldsymbol{s}}^{\alpha}\right)+\theta\left(\frac{\partial^{2} \hat{\psi}}{\partial \theta \partial \boldsymbol{\epsilon}^{e}}: \dot{\boldsymbol{\epsilon}}^{e}\right)}_{D_{\text {mech }}-H_{\theta}}-\nabla \cdot \boldsymbol{q}+r_{\theta},
$$

which can be written in a simplified expression by Simo and Miehe [85],

$$
c_{v} \dot{\theta}=\left[D_{\text {mech }}-H_{\theta}\right]-\nabla \cdot \boldsymbol{q}+r_{\theta} .
$$

Here $c_{v}$ is the specific heat per unit volume at constant deformation; $D_{\text {mech }}$ denotes the contribution to the dissipation due to pure mechanical load and/or thermal flow, which may be consistent in the form of $\beta \sigma: \dot{\boldsymbol{\epsilon}}^{p}$ with $\beta$ the TaylorQuinney coefficient; $H_{\theta}$ is the non-dissipative (latent) thermoelastic structural heat or cooling.

\subsection{A specific free energy functional}

We derive the explicit expressions for the balance equations by choosing the following stored free energy functional:

$$
\psi=\hat{\psi}^{e}\left(\boldsymbol{\epsilon}^{e}, \theta, \underline{d}\right)+\hat{\psi}^{p}(\underline{s}, \underline{d} ; \theta)+\hat{\psi}^{\mathrm{c}}(\underline{d}, \nabla \underline{d}, \underline{\omega})+\hat{\psi}^{\theta}(\theta) .
$$

The elastic part $\hat{\psi}^{e}$ is composed of the thermoelastic strain energy $\left(w^{e}\right)$ considering the thermal expansion [71,72,84,86-88], which is multiplied by the degradation function $g(\underline{d})$ for damage evolution:

$$
\hat{\psi}^{e}=g(\underline{d}) w^{e}\left(\boldsymbol{\epsilon}^{e}, \theta\right) \text { with } w^{e}\left(\boldsymbol{\epsilon}^{e}, \theta\right)=\frac{1}{2} \boldsymbol{\epsilon}^{e}: \mathbb{C}^{e}: \boldsymbol{\epsilon}^{e}-3 \alpha K\left(\theta-\theta_{0}\right) \operatorname{tr} \boldsymbol{\epsilon}^{e} .
$$

Firstly, the degradation function $g(\underline{d})$ for multiple phase-field can be assumed to have a simple form [54,55] as,

$$
g(\underline{d})=(1-k) \prod_{i}\left(1-d_{i}\right)^{2}+k
$$


This function has been chosen such that $g^{\prime}\left(d_{i}=1\right)=0$ to guarantee that the strain energy density function takes a finite value as the domain is locally cracked. The small parameter $k \ll 1$ is introduced for maintaining the wellposedness of the problem for partially broken part of the domain (cf. Heister et al. [89], Nguyen et al. [54,55]). Next $\mathbb{C}^{e}$ denotes the fourth-order elasticity tensor, which may constitute the isotropic or cubic symmetry for crystalline materials. $\alpha$ is the thermal expansion coefficient for isotropic behavior, $K$ is the bulk modulus, and $\theta_{0}$ denotes a fixed reference temperature. To avoid crack propagation under compression, the elastic strain can be further decomposed into a positive part $w_{+}^{e}$ and negative part $w_{-}^{e}$ by the spectral decomposition or the volumetric and deviatoric split $[55,71,90,91]$.

$$
\hat{\psi}^{e}=g(\underline{d}) w_{+}^{e}+w_{-}^{e},
$$

where the description of $w_{+}^{e}$ and $w_{-}^{e}$ for crystalline materials will be revisited in the following section.

We then take the contribution of plastic work for crack growth into account to simulate ductile fracture behavior. The simple equation for the plastic work may have the form:

$$
\hat{\psi}^{p}=g(\underline{d})^{p}\left\langle w^{p}-w_{0}^{p}\right\rangle, \text { where } g(\underline{d})^{p}=g(\underline{d}) \text { and } w^{p}=\frac{1}{2} \sum_{\alpha} h\left(s^{\alpha}\right)^{2} .
$$

In our formulation, the plastic work is temperature-dependence due to the incorporation of temperature-dependent creep motion (see the viscoplastic flow rule in (60)). Nevertheless, we follow the treatment in [85] and assume that the free energy function is temperature-independent for simplicity. As such, the thermoelastic heating contains no latent plastic terms and is in fact identical to the Gough-Joule effect, as explained in Simo and Miehe [1992]. A more comprehensive treatment that considers the plastic contribution in thermo-plastic solids can be found in recent work by Aldakheel [70].

Here the degradation function for plastic work is assumed to be identical to that of the elastic work, and a plastic work threshold $w_{0}^{p}$ may be introduced for controlling the plastic deformation in ductile fracture (e.g., Borden et al. [91], Miehe et al. [71]). The angle bracket operator is defined as,

$$
\langle x\rangle= \begin{cases}0 & \text { if } x<0 \\ x & \text { if } x \geq 0\end{cases}
$$

The plastic work $\omega^{p}$ comes from the hardening contribution, which is consistent with the Taylor hardening in (58). Therefore, the thermodynamic force $g^{\alpha}$ power-conjugate to $\dot{s}^{\alpha}$ in (35) can be explicitly described as,

$$
g^{\alpha}=-\sum_{\alpha} h s^{\alpha} .
$$

The stored energy function $\hat{\psi}^{c}(\underline{d}, \nabla \underline{d}, \underline{\omega})$ describes the total anisotropic crack surface energy. The total crack density formulation is previously defined by (10) based on the diffusive crack topology using the multi-phase-field approximation (Section 2.3). By assuming the same fracture energy for each slip direction (or the same energy release rate, $G_{c}$ ), the total crack energy function can be expressed as,

$$
\hat{\psi}^{c}=G_{c} \sum_{i}\left[\frac{1}{2 l}\left(d_{i}\right)^{2}+\frac{l}{2} \omega_{i}:\left(\nabla d_{i} \otimes \nabla d_{i}\right)\right] .
$$

The purely thermal contribution on the stored energy function $\hat{\psi}^{\theta}$ constitutes the heat transfer, in which the equation of $\hat{\psi}^{\theta}$ may have a simple form as [71,84,86-88,92,93],

$$
\hat{\psi}^{\theta}=c_{v}\left[\left(\theta-\theta_{0}\right)-\theta \log \left(\theta / \theta_{0}\right)\right],
$$

where $c_{v}$ is the specific heat defined in (37). The specific heat is assumed to be a constant for single-crystal halite based on Urquhart and Bauer [3]. In addition, we simplify the thermal-mechanical-fracture coupling problems by assuming that the stored thermal energy is not affected by fracture (cf., Miehe et al. [71]).

Finally, the microscopic multiple phase-field evolution equation for each phase of (33) now becomes,

$$
2\left(1-d_{i}\right) \mathcal{H}_{i}+\frac{G_{c}}{l} d_{i}+G_{c} l \nabla \cdot\left(\omega_{i} \cdot \nabla d_{i}\right)=0,
$$


where $\mathcal{H}_{i}$ is the strain-history functional that governs the evolution of the irreversible crack propagation (cf. Nguyen et al. $[54,55])$. This functional may take the following form,

$$
\mathcal{H}_{i}=\max _{\tau \in[0, t]}\left\{\prod_{j \neq i}\left(1-d_{j}\right)^{2}\left[w_{+}^{e}+\left\langle w^{p}-w_{0}^{p}\right\rangle\right]\right\} .
$$

\section{Constitutive law}

In this section, we present the constitutive law to capture the anisotropic behavior of crystalline materials. We combine the multi-phase-field method and the single-crystal plasticity theory to replicate the damage-elasto-plastic behavior via the effective stress theory (e.g., Lemaitre [94], Simo and Ju [95], Ju [96], de Borst et al. [97]). The effective stress theory hypothesizes that there exists an effective stress space where local stresses are redistributed to the effective area such as the undamaged skeleton of the body, undamaged material micro-bonds, the vicinity of growing voids. In addition, we also employ the hypothesis of strain equivalence. This assumption states that the strain caused by applying the total stress on the actual damaged material is the same as the strain caused by applying the effective stress on the fictitious undamaged material. As a result, the local elastoplastic constitutive responses and the evolution of internal variables can be resolved in a decoupled manner. While this technique is often employed for coupling damage and plasticity models (e.g., de Borst and Verhoosel [60]), Choo and Sun [61] have recently used this technique to couple a pressure-dependent plasticity model and the phase-field fracture framework.

Recalling the specific free energy function, (40) and (41), with the constitutive relation (30) reaches that the Cauchy stress, or total stress, can be resolved as,

$$
\boldsymbol{\sigma}=\frac{\partial \hat{\psi}}{\partial \boldsymbol{\epsilon}^{e}}=g(\underline{d}) \hat{\boldsymbol{\sigma}} \quad \text { with } \hat{\boldsymbol{\sigma}}=\mathbb{C}^{e}: \boldsymbol{\epsilon}^{e}-3 \alpha K\left(\theta-\theta_{0}\right) \mathbf{1},
$$

where $\hat{\sigma}$ denotes the effective stress or damage effective stress. It should be noted that the term effective stress of this study is different from that of porous media, in which the effective stress indicates the stress of the solid skeleton when saturated or partially saturated with fluid. (e.g., Coussy [98], Sun [99], Sun et al. [100], Sun [101], Wang and Sun [102], Na and Sun [103,104], Choo and Borja [105], Choo et al. [106]). The stress update algorithm for the multi-phase-field approach coupled to crystal plasticity is described in Algorithm 1.

\subsection{Single-crystal elasticity}

In this study, the elastic response of single-crystal halite is described by the fourth-order elasticity tensor $\mathbb{C}^{e}$ as in (41) and (51). As a crystal structure having cubic symmetry, single-crystal halite can be represented by three elastic constants, $C_{11}, C_{12}$, and $C_{44}$, where the $x, y$, and $z$ axes are aligned with the crystallographic axes (e.g., Hirth and Lothe [107], Wachtman et al. [108]). Apart from the anisotropic features originated from the microstructure, the elastic behavior of halite depends on temperature, pressure, and loading conditions (e.g., Durand [109], Bartels and Schuele [110]). Therefore, the experimental set-up including sample preparation process is an integral part to estimate the elastic parameters. There have been many efforts to measure the elastic constants of natural rock salt. According to Birch [111], the ultrasonics, one of the dynamical methods, may be the best technique to estimate the elastic properties of halite including its aggregates. One of the pioneering works was proposed by Oliver and Pharr [112], in which the load-displacement data from indentation experiments is used for determining elastic modulus of materials. Here we assume that the effect of elastic anisotropy is minor compared to the effect of plastic and damage anisotropy, following the standard assumption in crystal plasticity [113]. A more accurate model may apply the Zener anisotropy factor (cf. Clayton [73] p. 559) to estimate how severe the assumption of elastic isotropy is for halite. The optimal estimation of material parameters via inverse problems or optimization procedure, and the calculation of Zener anisotropy factor for halite are out of the scope but will be considered in future work. The average bulk modulus $(K)$ and Poisson's ratio (v), obtained from single-crystal data in Carter and Norton [67], are presented in Table 1.

The thermoelastic strain energy $\left(w^{e}\right)$ in $(41)$ can be rewritten in terms of volumetric and deviatoric strain as,

$$
w^{e}=\frac{1}{2} K\left(\epsilon_{v}^{e}\right)^{2}+\mu\left(\boldsymbol{\epsilon}_{d}^{e}: \boldsymbol{\epsilon}_{d}^{e}\right)-3 \alpha K\left(\theta-\theta_{0}\right) \epsilon_{v}^{e}, \text { with } \boldsymbol{\epsilon}^{e}=\frac{1}{3} \epsilon_{v}^{e} \mathbf{1}+\boldsymbol{\epsilon}_{d}^{e},
$$


Table 1

Material properties of the specimens for the numerical simulations.

\begin{tabular}{llll}
\hline Parameters & Description & Value & Unit \\
\hline$K$ & Bulk modulus & 25.0 & $\mathrm{GPa}$ \\
$v$ & Poisson's ratio & 0.25 & - \\
$\tau$ & Critical resolved shear stress & $5.0 \times 10^{-3}$ & $\mathrm{GPa}$ \\
$h$ & Hardening parameter & $1.0 \times 10^{-1}$ & $\mathrm{GPa}$ \\
$G_{c}$ & Fracture energy & 1.15 & $\mathrm{~J} / \mathrm{m}^{2}$ \\
$l$ & Length scale & $1.0 \times 10^{-5}$ & $\mathrm{~m}$ \\
$\beta$ & Anisotropy factor & 40.0 & - \\
$Q$ & Activation energy & 14.0 & $\mathrm{kcal} / \mathrm{mol}$ \\
$R$ & Gas constant & $1.986 \times 10^{-3}$ & $\mathrm{kcal} / \mathrm{mol} / \mathrm{K}$ \\
$C_{0}$ & Shape factor & 1.0 & - \\
$p$ & Exponent parameter & 10.0 & - \\
$\alpha$ & Thermal expansion coefficient & $11.0 \times 10^{-6}$ & $1 / \mathrm{K}$ \\
$c_{v}$ & Specific heat & $2.0 \times 10^{6}$ & $\mathrm{~J} / \mathrm{m}^{3} / \mathrm{K}$ \\
$\kappa$ & Thermal conductivity & 2.0 & $\mathrm{~W} / \mathrm{m} / \mathrm{K}$ \\
\hline
\end{tabular}

where $K$ and $\mu$ are the elastic bulk and shear moduli, respectively, $\epsilon_{v}^{e}=\operatorname{tr} \epsilon^{e}$ is the volumetric strain, and $\boldsymbol{\epsilon}_{d}^{e}$ is the deviatoric strain tensor. By decomposing the elastic strain into volumetric and deviatoric contributions, we can further separate the thermoelastic strain energy as follows to prevent unrealistic crack propagation under compression:

$$
\left\{\begin{array}{l}
w_{+}^{e}:=\frac{1}{2} K\left\langle\epsilon_{v}^{e}\right\rangle_{+}^{2}+\mu\left(\boldsymbol{\epsilon}_{d}^{e}: \boldsymbol{\epsilon}_{d}^{e}\right)-3 \alpha K\left(\theta-\theta_{0}\right)\left\langle\epsilon_{v}^{e}\right\rangle_{+} \\
w_{-}^{e}:=\frac{1}{2} K\left\langle\epsilon_{v}^{e}\right\rangle_{-}^{2}-3 \alpha K\left(\theta-\theta_{0}\right)\left\langle\epsilon_{v}^{e}\right\rangle_{-}
\end{array} .\right.
$$

This additive volumetric-deviatoric split is proposed by Amor et al. [114], while the split of the compressive and tensile components via spectral decomposition can be found in Miehe et al. [45].

\subsection{Single crystal plasticity}

This section presents the stress update algorithm for single-crystal plasticity on effective stress space. The unified fully implicit return algorithm for both rate-independent and rate-dependent settings is implemented based on [41]. Within this framework, a multisurface-type model with the elastic domain is used as in [38], and the power-lawtype viscoplastic slip rate is introduced for single-crystal halite. This slip-rate equation takes temperature- and ratedependent effects into account to describe the dislocation creep. For the rate independent limit, the pseudo-inverse method based on the singular value decomposition (SVD) is used to obtain the pseudo-inverse of the Jacobian matrix in the constitutive equations (e.g., Anand and Kothari [40], Miehe and Schröder [41]).

Following (4), we first denote the homogeneous strain rate $\dot{\boldsymbol{\epsilon}}$ in a crystal and consider its additive decomposition into elastic $\left(\dot{\boldsymbol{\epsilon}}^{e}\right)$, plastic $\left(\dot{\boldsymbol{\epsilon}}^{p}\right)$, and thermal $\left(\dot{\boldsymbol{\epsilon}}^{\theta}\right)$ parts, respectively.

$$
\dot{\boldsymbol{\epsilon}}=\dot{\boldsymbol{\epsilon}}^{e}+\dot{\boldsymbol{\epsilon}}^{p}+\dot{\boldsymbol{\epsilon}}^{\theta} .
$$

The plastic component coming from the slips on crystallographic planes results in the following expression (cf. (5)):

$$
\dot{\boldsymbol{\epsilon}}^{p}=\sum_{\alpha} \dot{\gamma}^{\alpha} \boldsymbol{S}^{\alpha}
$$

Again, $\dot{\gamma}^{\alpha}$ and $S^{\alpha}$ denote the plastic slip rate and the symmetric part of the Schmid tensor on $\alpha$-slip system, respectively.

Now we present the stress update algorithm for a crystal with $2 N$ potentially active slip systems, $\mathcal{J}:=$ $\{1,2, \ldots, 2 N\}$. Considering the microforce balance equation (18) for the slip system and crystal plasticity theory (e.g., Miehe and Schröder [41], Borja and Rahmani [42], de Souza Neto et al. [37]), the yield condition can be 
expressed as a function of the Schmid resolved shear stress $\left(\tau^{\alpha}\right)$ and the critical resolved shear stress $\left(\tau_{Y}^{\alpha}\right)$ for each slip system. Here we set up the yield condition based on the effective stress and critical resolved shear stress (e.g., Qi and Bertram [115], Ekh and Runesson [59]),

$$
f^{\alpha}=\tau^{\alpha}-\tau_{Y}^{\alpha}, \text { where } \tau^{\alpha}=\hat{\boldsymbol{\sigma}}: \boldsymbol{S}^{\alpha} .
$$

Then a non-smooth convex elastic domain in the stress space can be defined as,

$$
\mathbb{E}=\left\{\left(\hat{\boldsymbol{\sigma}}, \tau_{Y}^{\alpha}\right) \mid f^{\alpha} \leq 0 \text { for } \alpha=1,2, \ldots, 2 N\right\} .
$$

The evolution of these resistances within a multislip deformation process is governed by the hardening equations,

$$
\dot{\tau}_{Y}^{\alpha}=h \sum_{\beta=1}^{2 N} \dot{\gamma}^{\beta} .
$$

The hardening of the yield surface is characterized by the simple Taylor hardening law that introduces only one additional material parameter, i.e., the plastic modulus $(h)$. Here we rewrite the plastic strain rate in Koiters's form for rate-dependent single-crystal plasticity as,

$$
\dot{\boldsymbol{\epsilon}}^{p}=\sum_{\alpha=1}^{2 N} \dot{\gamma}^{\alpha} \frac{\partial f^{\alpha}}{\partial \hat{\boldsymbol{\sigma}}}=\sum_{\alpha=1}^{2 N} \dot{\gamma}^{\alpha} \boldsymbol{S}^{\alpha},
$$

where the slip rate $\dot{\gamma}^{\alpha}$ satisfies a constitutive viscoplastic form under loading conditions to incorporate plastic flow by the dislocation creep motion in single-crystal halite as (cf. Wawersik and Zeuch [116], Miehe and Schröder [41], Hansen et al. [19]):

$$
\dot{\gamma}^{\alpha}=C_{0} \exp \left(-\frac{Q}{R \theta}\right)\left[\left(\frac{f^{\alpha+}}{\tau_{Y}^{\alpha}}+1\right)^{p}-1\right] .
$$

Here $C_{0}$ represents a fitting parameter; $Q$ is the activation energy; $R$ is the universal gas constant; $\theta$ is the absolute temperature; $p$ is a strain-rate-sensitivity exponent. The overstress function $f^{\alpha+}$ is defined by,

$$
f^{\alpha+}:= \begin{cases}f^{\alpha} & \text { if } f^{\alpha}>0 \\ 0 & \text { otherwise }\end{cases}
$$

The slip rate $\dot{\gamma}^{\alpha}$ takes the form of (60) such that the rate dependence can be introduced into the plastic constitutive responses [72]. Note that the dislocation creep equation obtained from the experiments (e.g., Wawersik and Zeuch [116]) is not originally intended for describing a microscopic slip-system response. Instead, it was used to describe the macroscopic response of a specimen. Nevertheless, we hypothesize that the creeping equation of the slip system takes a similar form. To minimize the discrepancy between simulated results and experimental data, we calibrate the material parameters by solving the inverse problems [117,118]. A brief explanation of this procedure is discussed in Appendix A.

The unified stress update algorithm on effective stress space is outlined for both rate-independent and ratedependent crystal plasticity frameworks. Algorithm 1 describes the elastic predictor phase on effective space, while the plastic corrector and determination of active slip systems are performed in Algorithm 2. In the rate-dependent case, the viscoplastic regularization of single-crystal plasticity for halite (60) is used. At the rate-independent limit, the slip rate $\dot{\gamma}^{\alpha}$ satisfies the classical Kuhn-Tucker conditions [41,42,64,119]:

$$
\dot{\gamma}^{\alpha} \geq 0, \quad f^{\alpha} \leq 0, \quad \dot{\gamma}^{\alpha} f^{\alpha}=0,
$$

for all $\alpha$. Furthermore, the inverse of the Jacobian (D) can be obtained directly if it is non-singular. If the loading rate is sufficiently low such that the response is approaching the rate-independent regime, the Jacobian (D) may become singular. In this case, the Moore-Penrose pseudo-inverse, which can be determined via the singular value decomposition (SVD), is used to compute the incremental plastic slip (cf. Anand and Kothari [40]). For brevity, we drop the subscript $n+1$ for variables pertaining to the time $t_{n+1}$.

In Algorithm 1 we assemble the trial active set by checking the yield conditions for each slip system (56) under the given temperature $\theta$. When the trial active set is empty, the material is in the purely elastic regime. If the trial active 


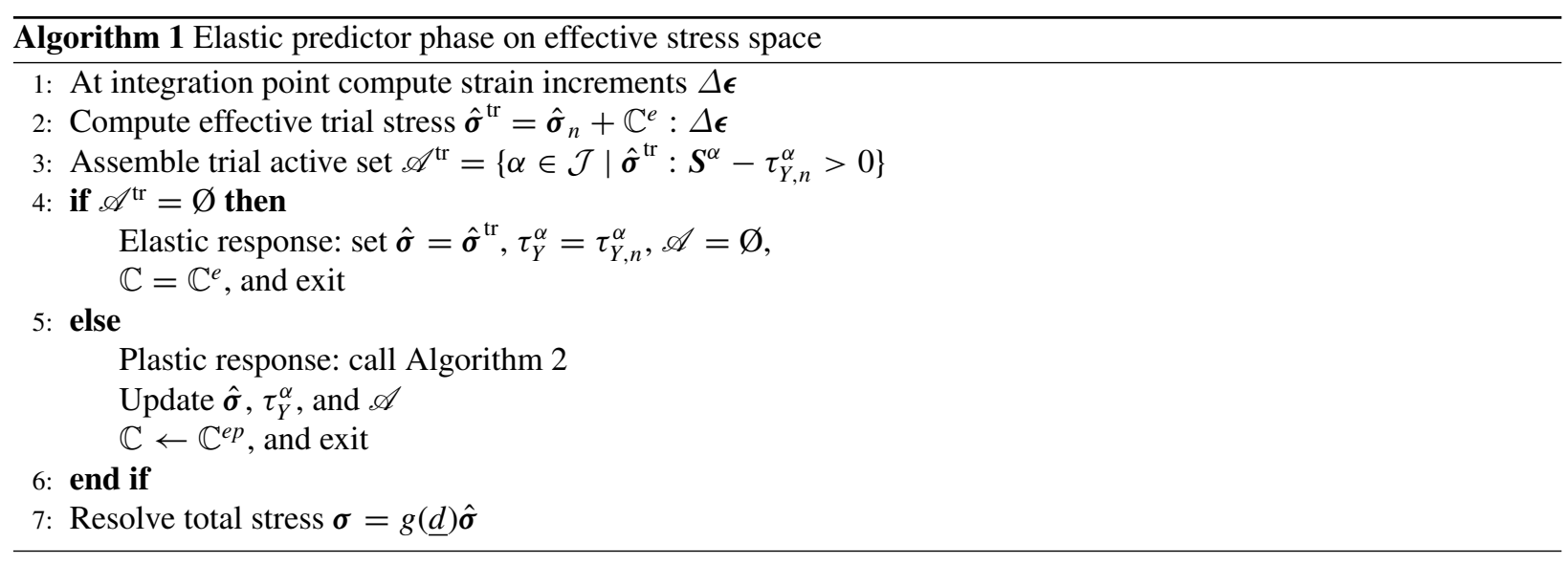

set contains any element, Algorithm 2 is triggered and the Newton iteration for the plastic response is performed. In this iteration step, the active slip system is successively checked and reassembled followed by two constraints. Firstly, we check $\gamma^{\alpha}$ in the trial active set whether each parameter violates the discrete loading conditions, (60) and (62), in the sense $\gamma^{\alpha} \leq 0$. If this is the case, we drop the slip system from the active set $\mathscr{A}$ and restart the local Newton iteration. Secondly, we check the yield conditions again for the slip systems, not in the current trial active set. We then reassemble the active set and restart the local Newton iteration by initializing $\gamma^{\alpha}=0$ for all $\alpha \in \mathscr{A}$. Otherwise, Algorithm 2 is terminated, and the updated parameters are forwarded to Algorithm 1 for the total stress calculation.

\section{Variational formulation}

This section describes a finite element formulation that combines the multi-phase-field and the crystal plasticity for modeling thermo-mechanical behaviors of single-crystal halite. We first derive the variational form of thermomechanical problems with multiple phase fields representing damage along preferential directions. This is followed by the description of a staggered scheme for solving the equilibrium (momentum and energy balances) and multiple phase-field equations in Section 5.

\subsection{Galerkin form}

We consider a domain $\mathcal{B}$ with its boundary $\partial \mathcal{B}$ composed of Dirichlet boundaries (solid displacement $\partial \mathcal{B}_{u}$, temperature $\partial \mathcal{B}_{\theta}$ ) and von Neumann boundaries (solid traction $\partial \mathcal{B}_{t}$, heat flux $\partial \mathcal{B}_{q}$ ) satisfying,

$$
\left\{\begin{array}{l}
\partial \mathcal{B}=\overline{\partial \mathcal{B}_{u} \cup \partial \mathcal{B}_{t}}=\overline{\partial \mathcal{B}_{\theta} \cup \partial \mathcal{B}_{q}} \\
\emptyset=\partial \mathcal{B}_{\boldsymbol{u}} \cap \partial \mathcal{B}_{t}=\partial \mathcal{B}_{\theta} \cap \partial \mathcal{B}_{q} .
\end{array}\right.
$$

The prescribed boundary conditions (Dirichlet and von Neumann boundary conditions) read,

$$
\left\{\begin{array}{rll}
\boldsymbol{u}=\overline{\boldsymbol{u}} & \text { on } & \partial \mathcal{B}_{\boldsymbol{u}}, \\
\boldsymbol{\sigma} \cdot \boldsymbol{n}=\overline{\boldsymbol{t}} & \text { on } \partial \mathcal{B}_{t}, \\
\theta=\bar{\theta} & \text { on } \partial \mathcal{B}_{\theta}, \\
-\boldsymbol{n} \cdot \boldsymbol{q}=\bar{q} & \text { on } \partial \mathcal{B}_{\boldsymbol{q}}, \\
\nabla \underline{d} \cdot \boldsymbol{n}=0 & \text { on } \partial \mathcal{B},
\end{array}\right.
$$

where $\boldsymbol{n}$ is the outward unit normal on surface $\partial \mathcal{B}$. For model closure, the initial conditions are imposed as,

$$
\boldsymbol{u}=\boldsymbol{u}_{0}, \quad \theta=\theta_{0} \text { at } t=t_{0} .
$$




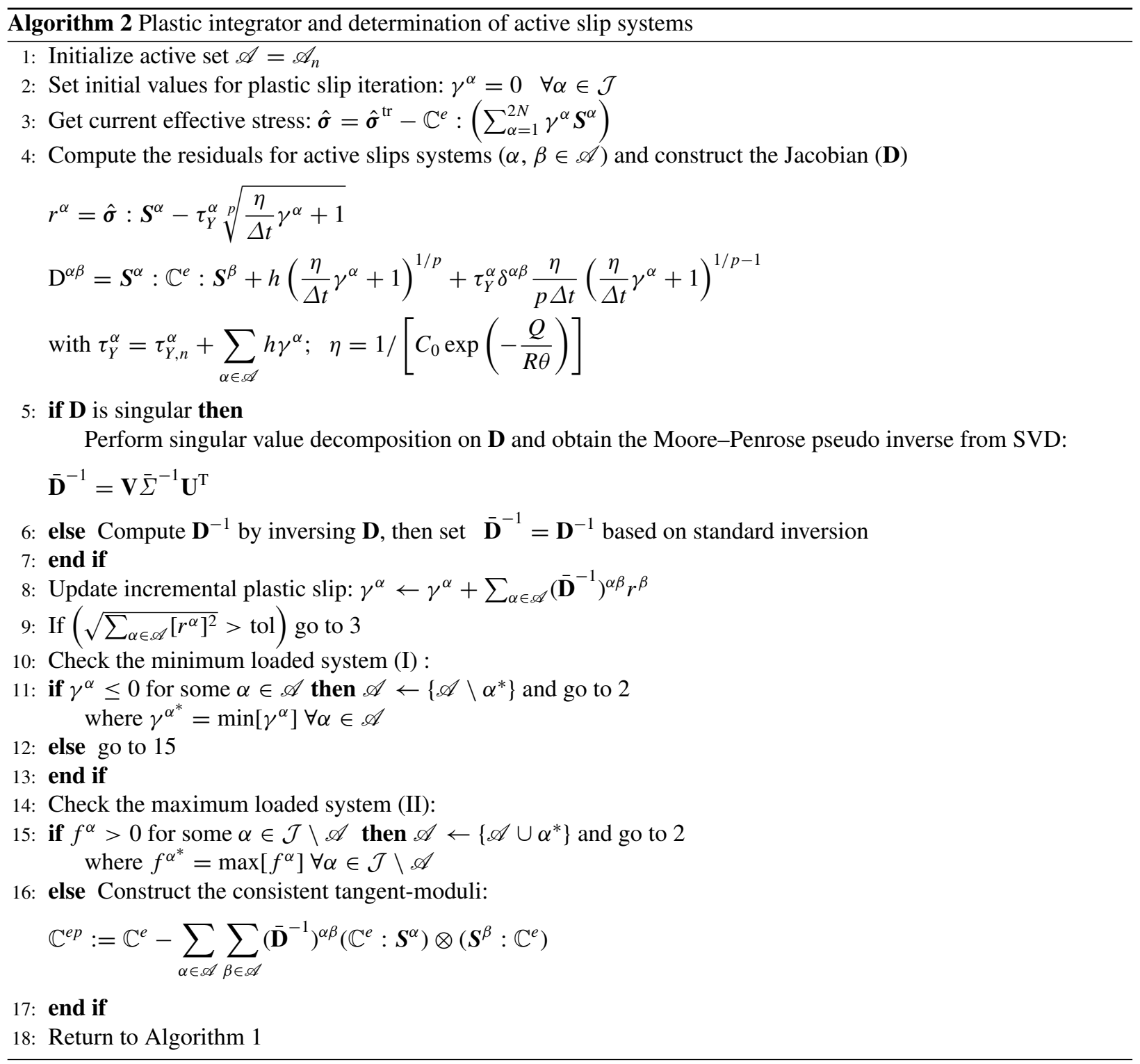

In addition, we consider the trial space for the weak form that reads,

$$
\begin{aligned}
V_{\boldsymbol{u}} & =\left\{\boldsymbol{u}: \mathcal{B} \rightarrow \mathbb{R}^{3}\left|\boldsymbol{u} \in\left[H^{1}(\mathcal{B})\right], \boldsymbol{u}\right|_{\partial \mathcal{B}_{u}}=\overline{\boldsymbol{u}}\right\}, \\
V_{\theta} & =\left\{\theta:\left.\mathcal{B} \rightarrow \mathbb{R}\right|_{\theta} \in H^{1}(\mathcal{B}),\left.\theta\right|_{\partial \mathcal{B}_{\theta}}=\bar{\theta}\right\}, \\
V_{\underline{d}} & =\left\{\underline{d}: \mathcal{B} \rightarrow \mathbb{R} \mid \underline{d} \in H^{1}(\mathcal{B})\right\}, \text { i.e., } \quad V_{d_{i}}=\left\{d_{i}: \mathcal{B} \rightarrow \mathbb{R} \mid d_{i} \in H^{1}(\mathcal{B})\right\} .
\end{aligned}
$$

Here $H^{1}$ denotes the Sobolev space of degree one. The number of $d_{i}$ depends on how we define preferential directions, which is consistent with the slip systems for single-crystal halite. The corresponding admissible spaces of variations are defined as,

$$
\begin{aligned}
V_{\eta} & =\left\{\boldsymbol{\eta}: \mathcal{B} \rightarrow \mathbb{R}^{3}\left|\boldsymbol{\eta} \in\left[H^{1}(\mathcal{B})\right], \eta\right|_{\partial \mathcal{B}_{u}}=\mathbf{0}\right\} \\
V_{\psi} & =\left\{\psi:\left.\mathcal{B} \rightarrow \mathbb{R}\right|_{\psi} \in H^{1}(\mathcal{B}),\left.\psi\right|_{\partial \mathcal{B}_{\theta}}=0\right\}, \\
V_{\underline{\phi}} & =\left\{\underline{\phi}: \mathcal{B} \rightarrow \mathbb{R} \mid \underline{\phi} \in H^{1}(\mathcal{B})\right\} \text { i.e., } \quad V_{\phi_{i}}=\left\{\phi_{i}: \mathcal{B} \rightarrow \mathbb{R} \mid \phi_{i} \in H^{1}(\mathcal{B})\right\} .
\end{aligned}
$$


Therefore, the weighted residual statements of the balance of linear momentum, energy, and phase-field are: Find $\boldsymbol{u} \in V_{\boldsymbol{u}}, \theta \in V_{\theta}$, and $\underline{d} \in V_{\underline{\phi}}$ such that all $\boldsymbol{\eta} \in V_{\eta}, \psi \in V_{\psi}$, and $\underline{\phi} \in V_{\underline{\phi}}$,

$$
G(\boldsymbol{u}, \theta, \underline{d}, \boldsymbol{\eta})=H(\boldsymbol{u}, \theta, \underline{d}, \psi)=\underline{L}(\boldsymbol{u}, \theta, \underline{d}, \underline{\phi})=0 .
$$

Consider the governing equations given by (16), (37), and (49). Through the standard weighted residual procedure, we obtain the variational equations as,

$$
\begin{aligned}
& G: V_{\boldsymbol{u}} \times V_{\theta} \times V_{\underline{d}} \times V_{\eta} \rightarrow \mathbb{R}, \\
& G(\boldsymbol{u}, \theta, \underline{d}, \boldsymbol{\eta})=\int_{\mathcal{B}} \nabla \boldsymbol{\eta}: \boldsymbol{\sigma} d V-\int_{\partial \mathcal{B}} \boldsymbol{\eta} \cdot \overline{\boldsymbol{t}} d \Gamma=0, \\
& H: V_{\boldsymbol{u}} \times V_{\theta} \times V_{\underline{d}} \times V_{\psi} \rightarrow \mathbb{R}, \\
& H(\boldsymbol{u}, \theta, \underline{d}, \psi)=\int_{\mathcal{B}} \psi c_{v} \dot{\theta} d V-\int_{\mathcal{B}} \psi\left[\sum_{\alpha}\left(\pi^{\alpha} \dot{\gamma}^{\alpha}+g^{\alpha} \dot{s}^{\alpha}\right)\right] d V-\int_{\mathcal{B}} \psi\left(3 \alpha K \mathbf{1}: \dot{\boldsymbol{\epsilon}}^{e}\right) \theta d V \\
& +\int_{\mathcal{B}} \nabla \psi \cdot \kappa \nabla \theta d V-\int_{\partial \mathcal{B}} \psi \bar{q} d \Gamma=0 \\
& \underline{L}: V_{\boldsymbol{u}} \times V_{\theta} \times \underline{V_{d}} \times V_{\underline{\phi}} \rightarrow \mathbb{R}, \\
& L_{i}\left(\boldsymbol{u}, \theta, d_{i}, \phi_{i}\right)=\int_{\mathcal{B}} \phi_{i}\left[2\left(1-d_{i}\right) \mathcal{H}_{i}\right] d V+\int_{\mathcal{B}} \frac{G_{c}}{l}\left[\phi_{i} d_{i}+l^{2} \nabla \phi_{i} \cdot \omega_{i} \cdot \nabla d_{i}\right] d V,
\end{aligned}
$$

where $\mathcal{H}_{i}$ is previously defined by (50).

\section{Operator-split solution strategies}

Due to nonlinearity and path-dependence nature of the proposed model, linearizing the system of equations is necessary if an implicit solver is used. In this work, the system of equations is multi-physical. As a result, this system of equations can be solved either in a monolithic or operator-split manner [120,121]. As previous numerical experiments with single phase-field problems show that the operator splitting approach may potentially be more robust (e.g., Miehe et al. [45], Heister et al. [89]), we propose a semi-implicit iterative strategy. In this procedure, the multiple phase fields are advanced followed by the thermo-mechanical solver, which updates the displacement and temperature fields together. These sub-systems are iteratively updated until all the residuals are below the tolerance.

In the proposed operator-split setting, the crack driving force $\mathcal{H}_{i}(50)$ is fixed while the multiple phase-fields are updated. As a result, the split multi-phase-field incremental problem becomes linear. The schematic of solution strategies is summarized as follows:

$$
\left[\begin{array}{c}
\boldsymbol{u}_{n} \\
\theta_{n} \\
\underline{d}_{n}
\end{array}\right] \underbrace{\stackrel{\mathcal{R}(\boldsymbol{u}, \theta)=\mathbf{0}}{\longrightarrow}\left[\begin{array}{c}
\boldsymbol{u}_{n+1} \\
\theta_{n+1} \\
\underline{d}_{n}=0
\end{array}\right]}_{\text {Iterative solver }} \overbrace{\frac{\mathcal{R}(\underline{d})=\underline{0} \text { with } \underline{\mathcal{H}}_{n+1}}{\delta \boldsymbol{u}=0, \delta \theta=0}\left[\begin{array}{c}
\boldsymbol{u}_{n+1} \\
\theta_{n+1} \\
\underline{d}_{n+1}
\end{array}\right]}^{\text {Linear solver }},
$$

where $\mathcal{R}(\boldsymbol{u}, \theta)$ and $\mathcal{R}(\underline{d})$ are residuals expressed as follows:

$$
\begin{aligned}
& \mathcal{R}(\boldsymbol{u}, \theta):\left\{\begin{array}{l}
\int_{\mathcal{B}} \nabla \boldsymbol{\eta}: \boldsymbol{\sigma}_{n+1} d V-\int_{\partial \mathcal{B}} \boldsymbol{\eta} \cdot \overline{\boldsymbol{t}}_{n+1} d \Gamma, \\
\int_{\mathcal{B}} \psi c_{v}\left(\frac{\theta_{n+1}-\theta_{n}}{\Delta t}\right) d V-\int_{\mathcal{B}} \psi\left[\sum_{\alpha}\left(\pi_{n}^{\alpha}\left(\frac{\gamma_{n}^{\alpha}-\gamma_{n-1}^{\alpha}}{\Delta t}\right)+g_{n}^{\alpha}\left(\frac{s_{n}^{\alpha}-s_{n-1}^{\alpha}}{\Delta t}\right)\right] d V\right. \\
-\int_{\mathcal{B}} \psi\left[3 \alpha K \mathbf{1}:\left(\frac{\boldsymbol{\epsilon}_{n}^{e}-\boldsymbol{\epsilon}_{n-1}^{e}}{\Delta t}\right)\right] \theta_{n+1} d V+\int_{\mathcal{B}} \nabla \psi \cdot \kappa \nabla \theta_{n+1} d V-\int_{\partial \mathcal{B}} \psi \bar{q}_{n+1} d \Gamma,
\end{array}\right. \\
& \mathcal{R}\left(d_{i}\right):\left\{\int_{\mathcal{B}} \phi_{i}\left[2\left(1-d_{i_{n+1}}\right) \mathcal{H}_{i_{n+1}}\right] d V+\int_{\mathcal{B}} \frac{G_{c}}{l}\left[\phi_{i} d_{i_{n+1}}+l^{2} \nabla \phi_{i} \cdot \omega_{i} \cdot \nabla d_{i_{n+1}}\right] d V .\right.
\end{aligned}
$$


These equations are consistent with (73) to (75), in which the backward Euler method is used for time discretization for heat transfer. Under the iterative solver in (76), the solutions are advanced followed by Algorithm 3 . This procedure requires the consistent tangent $\delta \mathcal{R}$ of the operator-split sub-problem, which is described in the following section. To obtain the incremental updates of the multiple phase fields, the updated $\mathcal{H}_{i}$ from the displacement and temperature variables at time $t_{n+1}$ is incorporated into the linear phase field solver. We then update the phase-field variables as in (76). To simplify the implementation, the temporal discretization of plastic dissipation and structural heating, i.e., the term $D_{\text {mech }}-H_{\theta}$ in (38) is treated explicitly. As shown in previous works such as Wang and Sun [122,123,77], this semi-implicit approach can be effective if used properly. Finally, it should be noticed that one may choose other partition strategies to solve the same system of equations. For instance, the thermo-mechanical problem can also be solved using an isothermal or adiabatic approach. The exploration of different partition strategies and the construction of the proper pre-conditioners are important subjects but are out of the scope of this study.

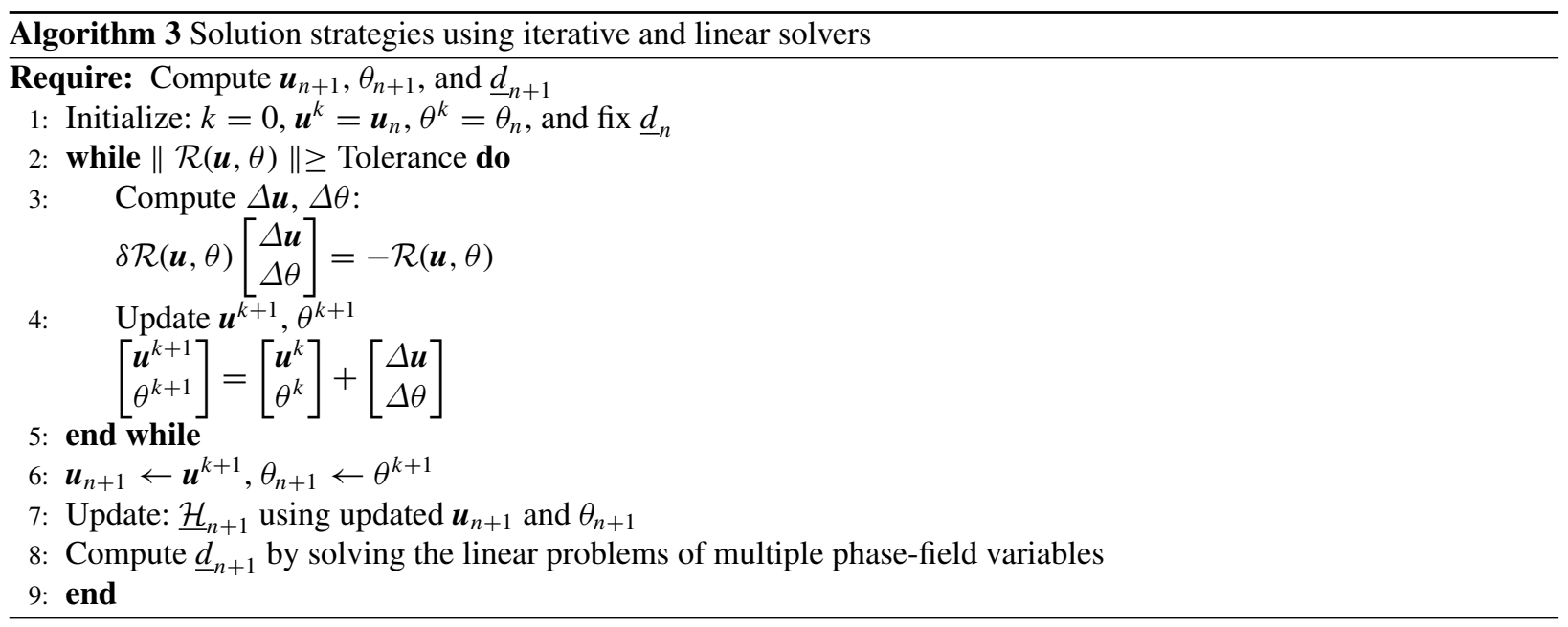

\subsection{Linearization for the staggered algorithm}

To obtain the numerical solution of the thermo-mechanical problems implicitly, the linearization of residuals of the governing equations is required. Due to the usage of the iterative sequential solver, the operator-split residual will only update one set of unknowns within one split iterative step. Hence the linearization is only consistent numerically for the staggered solver but not for the monolithic counterpart. With this in mind, we first describe the linearization of the balance of linear momentum with respect to the displacement and temperature as follows:

$$
\delta G(\boldsymbol{u}, \theta, \underline{d}, \boldsymbol{\eta})=\int_{\mathcal{B}} \nabla \boldsymbol{\eta}: \mathbb{C}: \delta \boldsymbol{u} d V-\int_{\mathcal{B}} \nabla \cdot \boldsymbol{\eta}[g(\underline{d}) 3 \alpha K] \delta \theta d V,
$$

in which $\mathbb{C}=g(\underline{d}) \mathbb{C}^{e p}$ can be obtained from the constitutive laws based on the effective stress concept (Algorithms 1 and 2). Likewise, the linearization of energy balance equation can be derived as follows:

$$
\begin{aligned}
\delta H(\boldsymbol{u}, \theta, \underline{d}, \psi)= & \int_{\mathcal{B}} \psi c_{v} \delta \theta d V-\int_{\mathcal{B}} \psi \delta\left[\sum_{\alpha}\left(\pi^{\alpha} \dot{\gamma}^{\alpha}+g^{\alpha} \dot{s}^{\alpha}\right)\right] d V-\int_{\mathcal{B}} \psi \delta\left(3 \alpha K \mathbf{1}: \dot{\boldsymbol{\epsilon}}^{e}\right) \theta d V \\
& -\int_{\mathcal{B}} \psi\left(3 \alpha K \mathbf{1}: \dot{\boldsymbol{\epsilon}}^{e}\right) \delta \theta d V+\int_{\mathcal{B}} \nabla \psi \cdot \kappa \delta(\nabla \theta) d V .
\end{aligned}
$$

We note that the plastic dissipation and structure heating terms in the energy balance equation (38) can be considered as a source term due to internal mechanical work. The consistent linearization of these terms for full implicit calculations requires complicated computations unless we employ automatic differentiation tools (e.g., Albany [124-126]). We, therefore, adopt the semi-implicit scheme for the energy balance equations by combining the backward Euler and forward Euler schemes (77). This procedure leads to the following tangent for the energy balance equation,

$$
\delta H(\boldsymbol{u}, \theta, \underline{d}, \psi)=\int_{\mathcal{B}} \psi c_{v} \delta \theta d V-\int_{\mathcal{B}} \psi\left(3 \alpha K \mathbf{1}: \dot{\boldsymbol{\epsilon}}^{e}\right) \delta \theta d V+\int_{\mathcal{B}} \nabla \psi \cdot \kappa \delta(\nabla \theta) d V .
$$


For completeness, we include the linearization of the multiple phase-field equations as follows:

$$
\delta L_{i}\left(\boldsymbol{u}, \theta, d_{i}, \phi_{i}\right)=\int_{\mathcal{B}}-2 \phi_{i} \mathcal{H}_{i} \delta d_{i} d V+\int_{\mathcal{B}} \frac{G_{c}}{l}\left[\phi_{i} \delta d_{i}+l^{2} \nabla \phi_{i} \cdot \omega_{i} \cdot \delta\left(\nabla d_{i}\right)\right] d V .
$$

Note again that this equation is for phase-field variable $d_{i}$, and other phase-field variables exhibit identical expressions.

\section{Numerical examples}

We present numerical examples to validate and evaluate the applicability of the proposed combined multi-phasefield crystal plasticity model for single-crystal halite. Firstly, plane strain (2D) tests are used to investigate influence of different orientations of the slip system on anisotropic behavior of halite. In particular, we compare the stress-strain curves, plastic slips, and crack phase fields obtained from multiple simulations with different relative angles between the slip-system and the loading direction. Then, we conduct additional tests with different mechanical and thermal boundary and initial conditions to evaluate the thermo-mechanical coupling effects of halite. A two-dimensional tension test with an existing crack is set up to examine how rate-dependence affects the onset and propagation of anisotropic fracture. Finally, to provide an insight on how grain boundary toughness affects the macroscopic mechanical behaviors, we conduct three tensile loading simulations on bicrystals with the same crystal properties but with grain boundary exhibiting different critical energy release rates.

The material properties, such as stiffness, strength, thermal parameters, activation energy, of halite depend significantly on the environmental and loading conditions. In an idealized case in which experimental data are sufficient, a subset of material parameters can be determined from the first principle, while the rest of the material parameters are then inferred by solving inverse problems (e.g., Wang et al. [118], Wang and Sun [127], Liu et al. [117]). However, due to the complexity of the inverse problems that involve both crystal plasticity and anisotropic fracture, such an approach is not used but will consider in the future study. As an alternative, the material parameters used in this study are obtained from the various sources in the literature. Firstly, the elastic moduli and fracture energy of single-crystal halite are obtained from the previous study by Tromans and Meech [128]. The length scale $l$ in Table 1 is on the order of mesh size and is used as a regularization parameter [55,114,129]. Meanwhile, the critical resolved stress, hardening parameter, and creep parameters are obtained from Wenk et al. [130], Wawersik and Zeuch [116]. The thermal parameters including the specific heat, thermal conductivity, and thermal expansion coefficients of halite were studied by Urquhart and Bauer [3]. The material properties used in this study are summarized in Table 1. Note that some of these material parameters, such as thermal conductivity, heat capacity, hardening parameter, and activation energy, are strictly speaking temperature dependent [70]. For instance, experimental data in Urquhart and Bauer [3], Birch and Clark [131], Smith [132] have shown that the thermal conductivity of single-crystal halite may be reduced by half from -75 to $100{ }^{\circ} \mathrm{C}$ whereas the specific heat remains close to $2.0 \mathrm{MJ} / \mathrm{m}^{3} / \mathrm{K}$. Nevertheless, these measurements are highly dependent on the experimental settings and the discrepancies among different data sets are large compared to the statistical noise. As a result, due to the fact that the geological disposal for nuclear waste is expected to operate in a higher temperature range [1], and the lack of sufficient experimental data to incorporating the temperature dependence of all the aforementioned material parameters, the temperature dependence of these material parameters is not considered in this work.

The implementation of the numerical model leverages Geocentric, a massively parallel finite element code for geomechanics, which is built on the open-source finite element library deal.II $[133,134]$ interfaced with the p4est mesh handling library [135], and the Trilinos project [136]. This code base has been widely used in previous studies including multiphysics problems (e.g., Choo and Borja [105], White et al. [121], White and Borja [137,138], Na and Sun [104], Na et al. [48], Borja and Choo [139]).

\subsection{Effect of crystal orientations}

In this section, numerical examples are presented to investigate the anisotropic mechanical behavior of singlecrystal halite when subjected to loadings under different orientations of the slip system. Fig. 3 depicts the boundary conditions and the definition of Euler angles for rotating the slip system. For boundary conditions, the horizontal directions are constrained on both top and bottom surfaces of the sample. The bottom surface is further constraint along the vertical direction, and the displacement boundary condition is applied at the top surface to compress the sample. Note that the influence of confining pressure is not considered, but its effect considering the grain boundary 


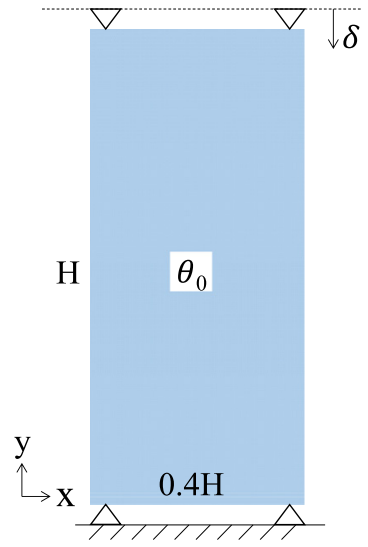

(a)

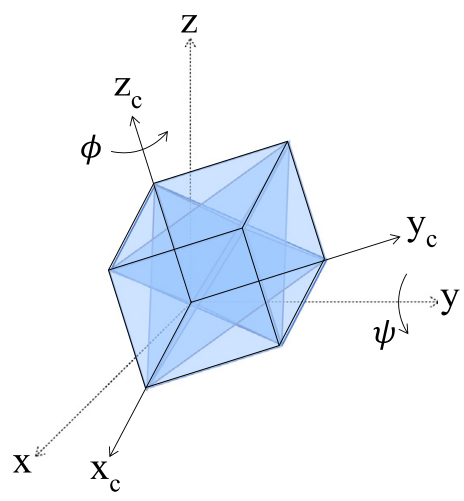

(b)

Fig. 3. Analysis of material anisotropy by changing the orientation of the slip system, (a) the set-up for plane strain compression test, (b) the definition of Euler angles $(\phi, \psi)$ in the three-dimensional plane (reproduced from Borja [65]) including the slip system of single-crystal halite (see each slip system in Fig. 2).

simulation will be covered in future study. As can be seen from Figs. 2 and 3, two cleavage planes (or slip planes) projected onto the 2D plane are used for directional information of multi-phase-field calculations. To be specific, unless changing orientations of the slip systems, each cleavage plane aligns to $45^{\circ}$ and $135^{\circ}$ directions onto $2 \mathrm{D}$ ( $x$-horizontal, $y$-vertical) plane, respectively. While such a simplified treatment can be physically reasonable in a plane strain idealized setting, a more comprehensive modeling effort should consider the non-coaxial cases. For instance, fracture planes that are more likely to fail, such as $\{100\}$ (cf. Wenk et al. [130]), can be associated with one of the phase fields. This improvement will be considered in future studies. We note that the full set of $\{110\}\langle 1 \overline{1} 0\rangle$ slip systems is considered for dislocation slip in crystal plasticity calculations, while the two slip planes are assumed to be aligned with the fracture planes.

For halite or other FCC crystals undergoing plane deformation, it is not essential to consider the whole set of the slip systems in the crystal plasticity model if the planar double-slip model is used [37,140]. This simplified model has been introduced in the pioneering study reported in Rashid and Nemat-Nasser [140] where only two "effective" slip systems whose slip and normal directions lie on the plane of loading. In this work, we follow the notation in Borja and Wren [39], Borja and Rahmani [42], Borja [65] and use two Euler angles, $(\phi, \psi)$, to describe the orientation of the crystal axes relative to the fixed reference system. We firstly use the default orientation (i.e., the slip orientations as in Fig. 2), and then set $\phi$ to $10^{\circ}$ and $30^{\circ}$, respectively, to rotate the slip system on the $x-y$ plane. Due to the constraint at the top and bottom surfaces, we obtain the localized plastic and damage zones without adding other conditions (e.g., geometric or material defects).

Four thousand bilinear quadrilateral finite elements are used to discretize the domain with the height $H=1.0 \mathrm{~mm}$. The displacement, temperature, and multiple phase fields are interpolated by the same basis functions. As a result, there are 12,423 displacement degree of freedoms (DOFs), 41,141 temperature DOFs and 8282 phase-field DOFs (4141 for each phase-field) before applying the essential boundary conditions. Under three different orientations, $(\psi$ is fixed at $0^{\circ}$; with $\phi=0^{\circ}, 10^{\circ}$, and $30^{\circ}$, respectively), we observe the significant influence of material anisotropy on the mechanical responses without introducing any defects or inhomogeneity. The constraint boundary condition with material anisotropy facilitates the different responses and patterns of plastic and damage behaviors. In these simulations, the reference temperature, or the initial temperature condition is set to $25^{\circ} \mathrm{C}$ for all three test cases. The top boundary is moving downward during the simulation with the strain rate of $1.0 \times 10^{-5} / \mathrm{s}$.

To analyze the mechanical responses under plane strain tests, the differential stress (the difference between the most and least compressive principal stresses) vs. axial strain curves obtained from specimens of different orientations are presented in Fig. 4. Although little difference is observed in the stress-strain curves obtained from $\phi=0$ and $\phi=$ $10^{\circ}$ cases, the resultant plastic slip patterns in Fig. 5 clearly show the anisotropy of the plastic and damage responses. When Euler angle $\phi$ is within the range from 0 to around $10^{\circ}$, the damage is localized at the boundaries. In the case 


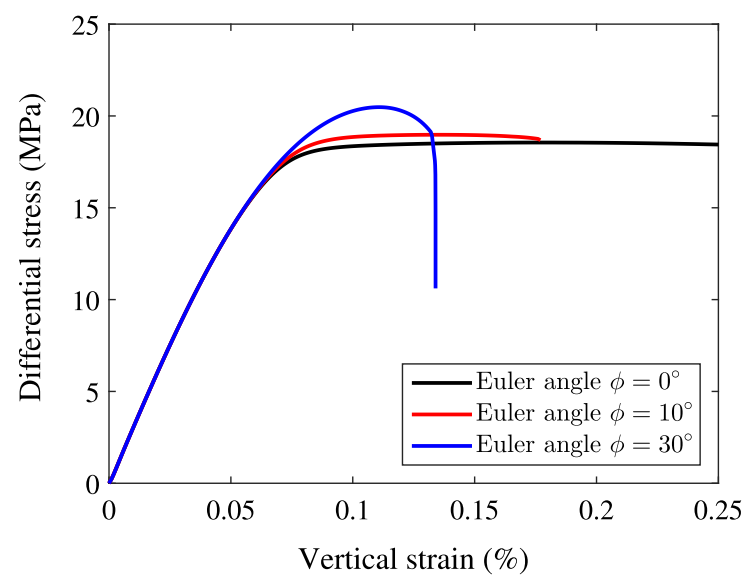

Fig. 4. Stress-strain curve of single-crystal halite with different orientations of the slip-system (the Euler angle $\psi$ is fixed $0^{\circ}$ while changing $\phi=$ $0^{\circ}, 10^{\circ}$, and $30^{\circ}$, respectively).

of $\phi=30^{\circ}$, however, we observe the localized damage zone in the center of the domain, not the edges. Furthermore, the plastic slip is also concentrated in accordance with the damaged zone. These patterns are consistent with the stress-strain curve in Fig. 4, which suggests the brittle fracture behavior. The temperature field for the effect of energy dissipation converging to heat is not presented, which has a minor role to influence the mechanical behavior of halite.

\subsection{Thermal effect on anisotropic creeping}

We conduct the plane strain compression tests with different boundary conditions to analyze the thermal effect on halite. Firstly we design the boundary conditions as depicted in Fig. 6. The left boundary is fixed along the horizontal direction, and the bottom surface is fixed against the vertical direction. The moving boundary with a constant strain rate $\left(1.0 \times 10^{-5} / \mathrm{s}\right)$ applies the loading on the top surface as in the previous numerical example. Under the same mechanical loading condition, we set three different initial reference temperatures on the sample domain: $25^{\circ} \mathrm{C}, 50{ }^{\circ} \mathrm{C}$, and $90{ }^{\circ} \mathrm{C}$, respectively. In these cases the same Euler angles $\psi=0^{\circ}$ and $\phi=70^{\circ}$ are used. Under different equilibrium temperature conditions, the mechanical responses including fracture and plastic behavior are evaluated.

First of all, we compare the mechanical responses under different temperature conditions using the differential stress and vertical strain curves in Fig. 6. In these cases the ductile fracture behaviors are observed while the plastic behaviors differ due to the difference in initial temperatures. Our crystal plasticity model adopts the creep behavior of halite that constitutes the temperature, which is essential in analyzing salt problems. Although the calibration has not been completed for temperature dependence and the fracture initiated on the sample, the influence of temperature on the mechanical behavior of halite is clearly identified. Furthermore, we present the damage and plastic patterns at the same vertical strain, $0.23 \%$ in Fig. 7. Followed by the stress-strain curves in Fig. 6, the clear fracture initiated from the top left edge is observed when the temperature is $25^{\circ} \mathrm{C}$. The plastic zones including the localized region can be further identified. As the initial temperature increases, the less damage is observed at the same displacement, which can be easily expected from the stress-strain curves. Interestingly, the more plastic slip is concentrated at the right bottom area of the domain as the temperature increases. This indicates that the temperature may further influence the patterns of plastic zones under the same mechanical loading condition.

We then design another boundary value problem to mimic the condition of a storage room in a geological repository subjected to temperature gradient due to the decay of the heat-generating nuclear waste. This problem is adopted from the facility of the Waste Isolation Pilot Plant (WIPP) report that investigates the room closure (cf. Reedlunn [141]). Because the field problem requires the modeling of large volume of domains composed of polycrystalline salt and other impurities, the single-crystal model is not directly applicable. Instead, we introduce a highly simplified problem which focuses on capturing the thermal-induced deformation of a single crystal on the wall of a room in the repository facilities as shown in Fig. 8(a). This room resembles a tunnel with a square shape section as depicted in Fig. 8(b). 

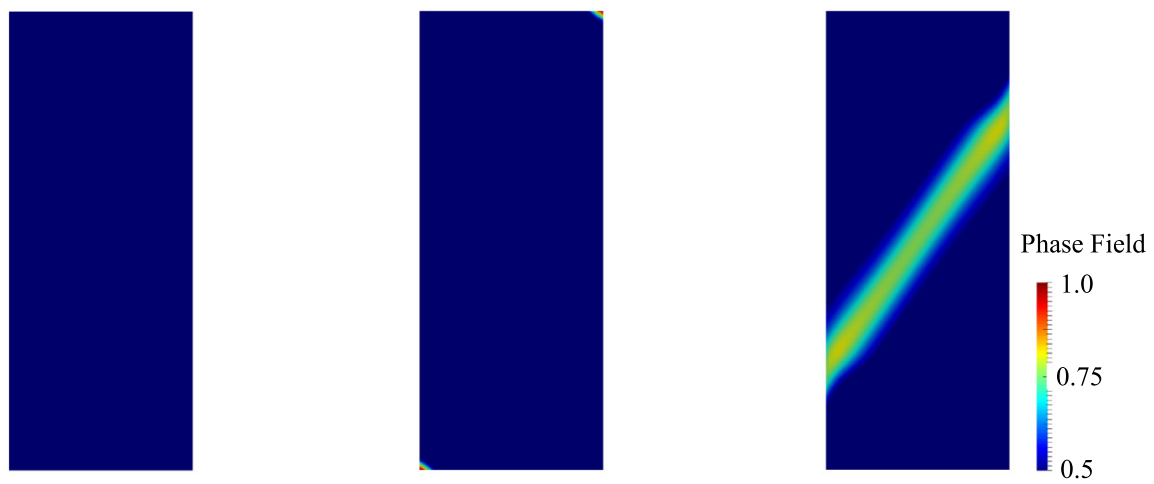

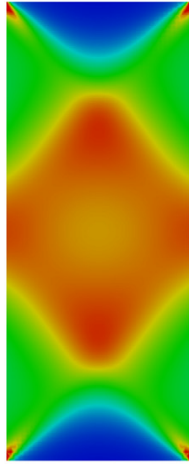

Rotation $\varphi=0^{\circ}$

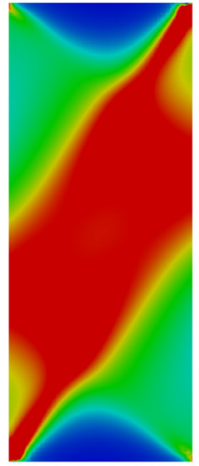

Rotation $\varphi=10^{\circ}$

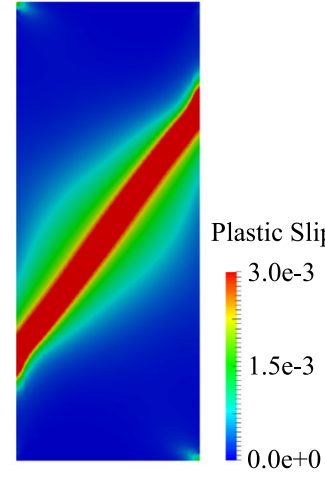

Rotation $\varphi=30^{\circ}$

Fig. 5. Phase-field values (top) and plastic slip results patterns (bottom) with different orientations of the slip-system to investigate the material anisotropy under plane strain compression test condition. The patterns are captured at the last numerical step of each case. Euler angle $\psi=0^{\circ}$ is fixed while $\phi$ varies 0,10 , and $30^{\circ}$, respectively.

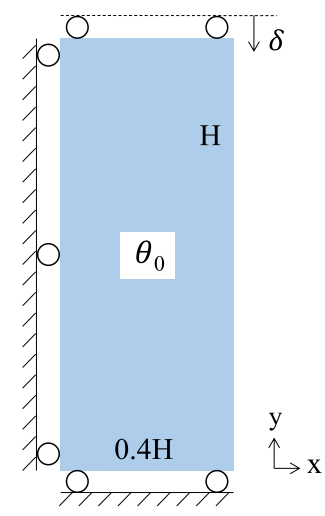

(a) Test set-up.

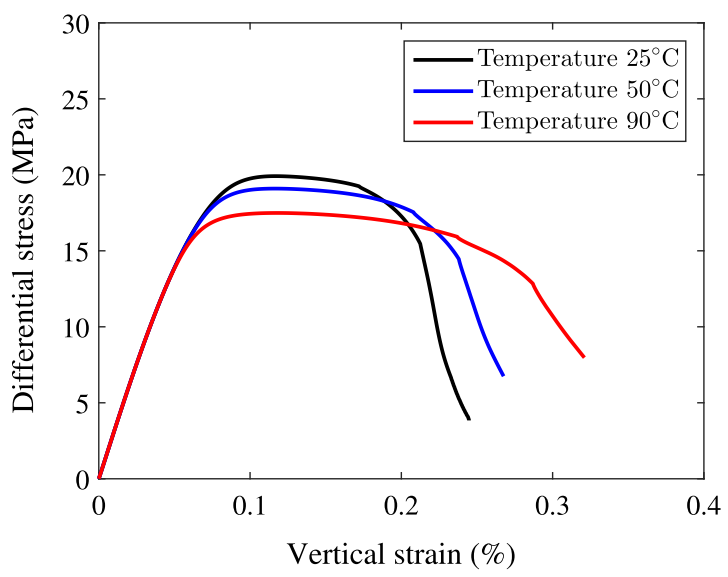

(b) Stress-strain curve.

Fig. 6. Analysis of temperature effect on mechanical responses, (a) the set-up for plane strain compression test, (b) stress-strain curves for each temperature condition $\left(\theta_{0}=25^{\circ} \mathrm{C}, 50^{\circ} \mathrm{C}\right.$, and $90^{\circ} \mathrm{C}$, respectively).

Once the construction of the room is completed, the disposed wastes fill the space. During the life cycle of the disposal facility, various amount of heat would generate as the radionuclides are decaying. Therefore, we consider an idealized 

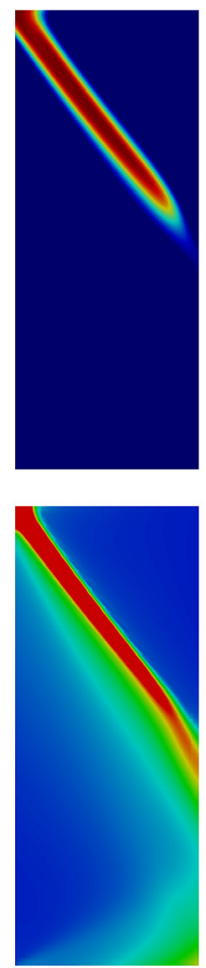

Temperature $25^{\circ} \mathrm{C}$
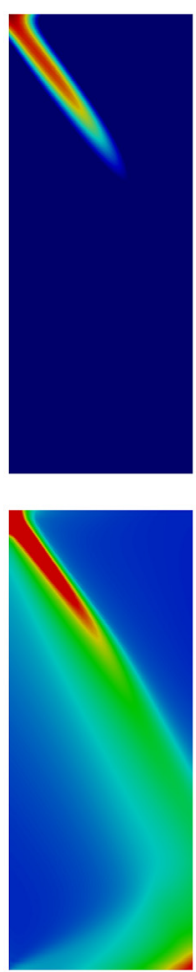

Temperature $50^{\circ} \mathrm{C}$
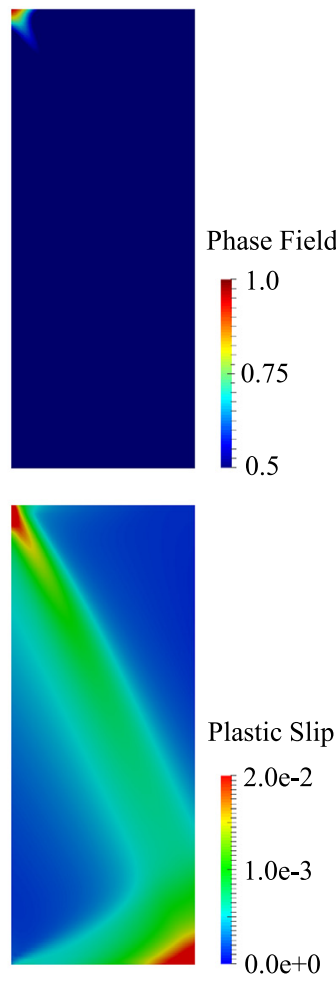

Temperature $90^{\circ} \mathrm{C}$

Fig. 7. Phase-field values (top) and plastic slip results (bottom) patterns with different initial temperature conditions at the same vertical strain, $0.23 \%$. The constant displacement loading is applied at the top, and the Euler angles are fixed with $\psi=0^{\circ}$ and $\phi=70^{\circ}$.

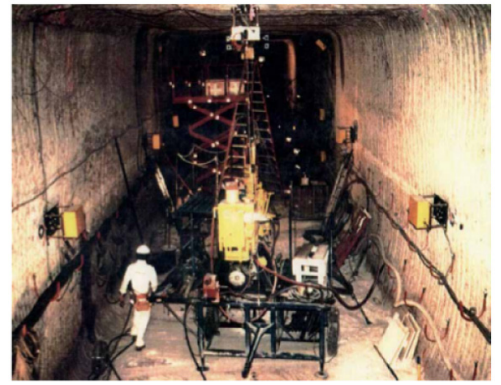

(a)

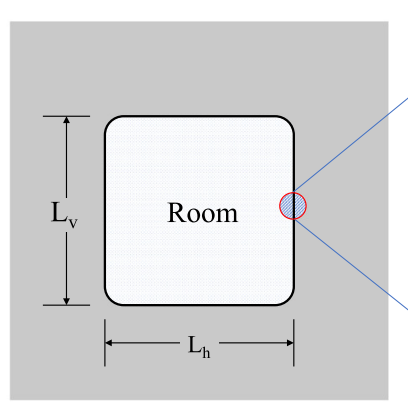

(b)

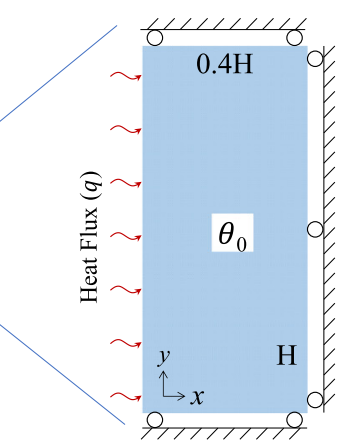

(c)

Fig. 8. The conceptual boundary value problem set-up for evaluating thermal influence on single-crystal halite, (a) a photo of room B (captured from Reedlunn, Munson et al. [141,142]), (b) the sections of rooms in WIPP (reproduced from Reedlunn [141]), and (c) the problem set-up. The dimension of a room section $\left(L_{v}\right.$ and $\left.L_{h}\right)$ varies on each room in the WIPP. The top, bottom, and right surfaces are insulated, and the initial temperature $\theta_{0}$ is set to $25^{\circ} \mathrm{C}$. The whole domain temperature is increased to $100{ }^{\circ} \mathrm{C}$ by applying constant heat flux $q$. The Euler angle is set to $\psi$ $=0^{\circ}$ and $\phi=70^{\circ}$.

situation in which single-crystal halite on the room surface is subjected to a heat source. As a result, the single-crystal halite is subjected to the boundary condition illustrated in Fig. 8(c). The Euler angles of the halite are set to $\psi=0^{\circ}$ and $\phi=70^{\circ}$. The top, bottom, and right surfaces are constrained and thermally insulated. Meanwhile, a heat flux is 


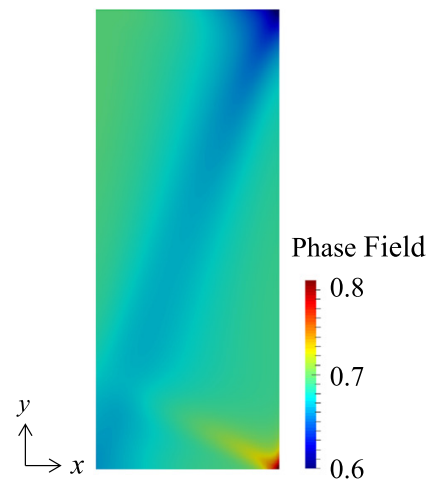

(a)

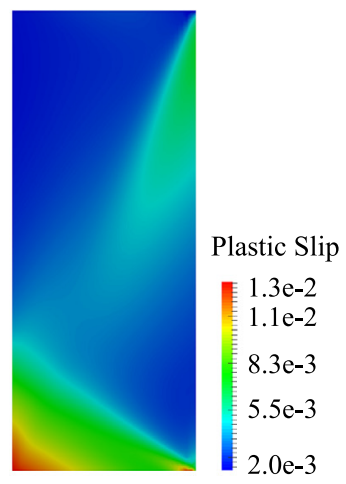

(b)

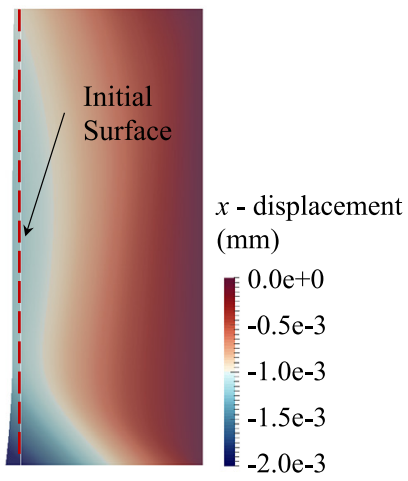

(c)

Fig. 9. The resultant patterns of (a) phase field for damages, (b) the plastic lip, and (c) the $x$ direction displacement (scaled by $\times 10)$ are illustrated when the overall temperature domain is increased up to $100^{\circ} \mathrm{C}$.

prescribed on the left surface. Initially, we set the temperature of the entire halite at $25^{\circ} \mathrm{C}$. Then, the heat flux leads to the temperature of the domain increases to $100^{\circ} \mathrm{C}$. As the single-crystal halite is subjected to heat flux, we observe the thermal induced damage-plastic responses.

Fig. 9 shows the phase field value, plastic slip, and the horizontal displacement in the deformed configuration. Due to the heat flux, the halite specimen deforms while accumulating plastic deformation and damage as in Fig. 9. While the deformation gradient is higher near the surface where the heat flux is prescribed, the phase field and plastic slip exhibit a very different pattern. Although the thermal expansion coefficient is isotropic, the existence of the slip system and cleavage planes leads to an anisotropic constitutive responses.

\subsection{Loading rate effect}

In this section, we conduct a simple tensile test on a halite specimen with a pre-existing crack. By changing the prescribed loading rates, we examine how the loading rate affects the interplays between the plastic deformation and the crack growth. The size of the square domain $L$ is $1 \mathrm{~mm}$, and the initial crack length is $0.5 \mathrm{~mm}$. The bottom surface of the specimen is constrained along both horizontal and vertical directions while the top surface is stretched vertically under a constant loading rate in each simulation. As demonstrated in Fig. 10(b), the ductile-brittle transition of halite under different loading rate is captured. Increasing the loading rate leads to higher peak differential stress and the material also behaves more ductile in the softening regime. This is consistent with the behavior of crystalline rock [143]. A closer look at the crack pattern and the distribution of the plastic strain also reveals that the loading rate affects the macroscopic responses as well as the anisotropic fracture and plastic deformation.

In particular, when the loading rate is slow $\left(\dot{\epsilon}=1.0 \times 10^{-7} / \mathrm{s}\right)$, the main crack tends to follow the cleavage plane directions (see the right figure in Fig. 11). As the strain rate is increased to $\dot{\epsilon}=1.0 \times 10^{-6} / \mathrm{s}$, (the $10 \dot{\epsilon}$ case in Fig. 11), a slight zig-zag pattern is observed. It appears that initially the crack follows the cleave plane but diverts the propagation direction due to loading conditions. This crack then follows another cleavage plane at the left edge of the domain. Finally, when the loading rate further increases (see the left figure in Fig. 11), the crack propagation appears to be straight and the damaged zone is also more diffusive than the low-strain-rate counterpart. This result is perhaps attributed to the different amount of energy dissipation due to creeping deformation. As the plastic flow of each slip system is highly sensitive to the loading rate (cf. (60)), the changes of loading rate may affect the magnitude and direction of the overall plastic flow. In the low strain rate case where the results resemble closely to the rateindependent crystal plasticity, the plastic flow is likely to be dominated by the fewer slip system, which causes plastic deformation appears to be more anisotropic. This in return changes the distribution of the driving force for each multiple phase field system in (50). As the multiple phase fields and plastic slip both evolve differently under different loading rates, this leads to the crack pattern appears to be more anisotropic in the low-strain-rate case. 


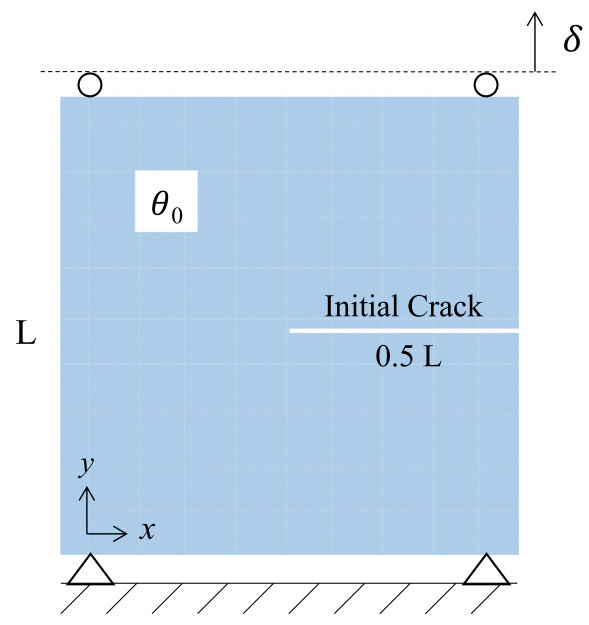

(a) Test set-up.

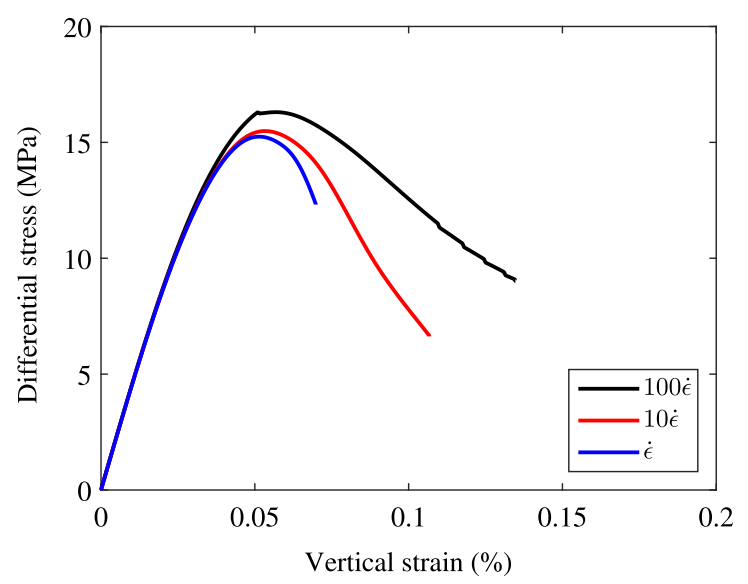

(b) Stress-strain curve.

Fig. 10. Analysis of the loading rate effect under two-dimensional tension test, (a) the set-up for boundary value problem, (b) stress-strain curve with different loading rates $\left(\dot{\epsilon}=1.0 \mathrm{e}^{-7} / \mathrm{s}\right)$. The initial temperature $\theta_{0}$ is set to $25^{\circ} \mathrm{C}$, and the Euler angles are set to $\psi=0^{\circ}$ and $\phi=0^{\circ}$.
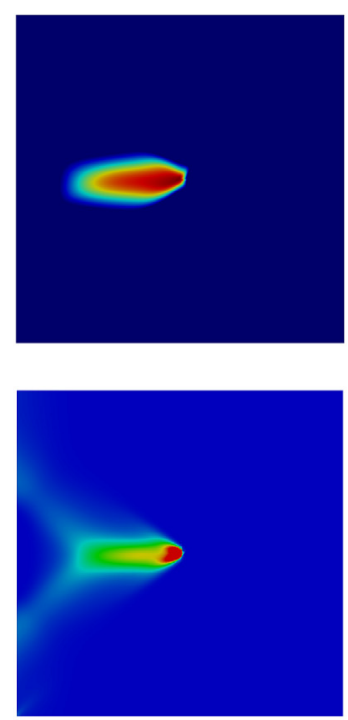

strain rate, $100 \dot{\epsilon}$
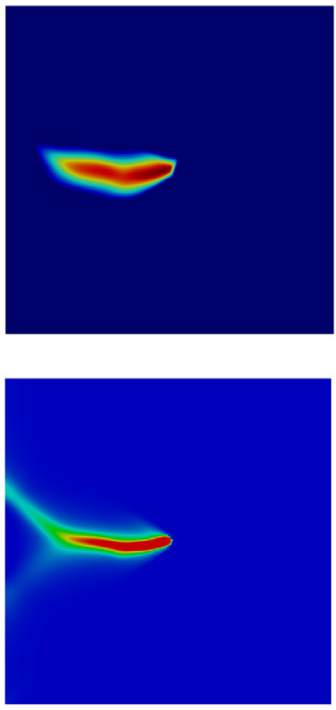

strain rate, $10 \dot{\epsilon}$
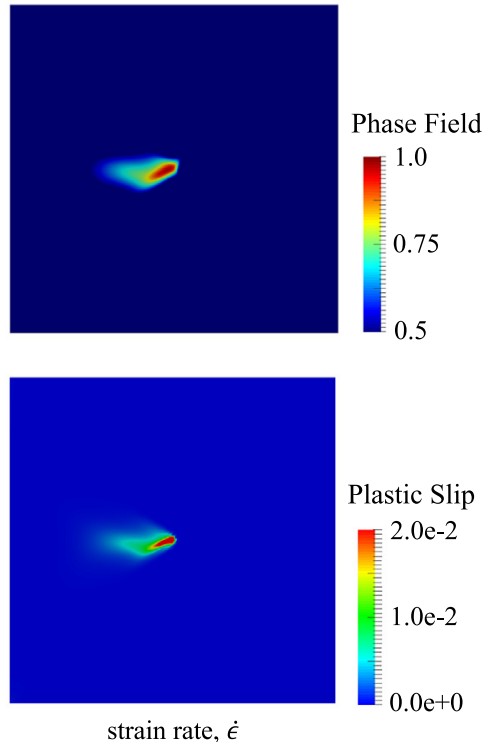

strain rate, $\dot{\epsilon}$

Fig. 11. Phase-field values (top) and plastic slip results (bottom) patterns with different loading rates are illustrated. The results are captured at the last numerical step of each simulation with the loading rate $\dot{\epsilon}=1.0 \mathrm{e}^{-7} / \mathrm{s}$.

\subsection{Crack propagation in a bicrystal halite}

In this final example, we simulate a tensile test conducted on a bicrystal domain to investigate the interaction between grain and grain boundary. Similar boundary value problems have been used previously in Oshima et al. [53] and Nguyen et al. [55] to model intragrain and grain boundary fractures. In this study, our major point of departure is (1) the introduction of crystal plasticity model to capture the plastic flow of the slip system inside the crystal and (2) the modeling of plastic slip of the grain boundary. To simplify the modeling effort, the grain boundary is approximated using a crystalline material with a finite thickness that has a single-cleavage plane of which the slip direction is aligned to the longitudinal direction of the grain boundary. The upshot of this treatment on grain boundary 


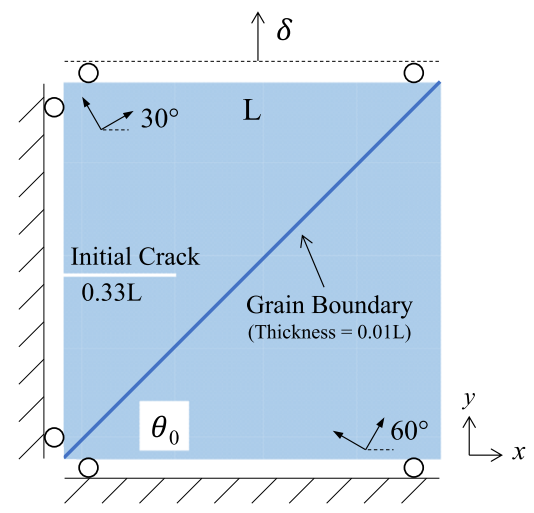

Fig. 12. The numerical set-up for a bicrystal is depicted to analyze the impact of the grain boundary fracture energy on crack propagations (reproduced from Oshima et al. [53]). The Euler angles $\phi$ are set to $30^{\circ}$ and $60^{\circ}$, respectively to left and right side grains. The single cleavage plane is assumed to the grain boundary with the direction of $45^{\circ}$ along the diagonal. The initial temperature is set to $25^{\circ} \mathrm{C}$, and the fracture energies of the grain boundary are adopted $86 \%, 75 \%$, and $50 \%$ of the grain fracture energy, respectively.

leads to a much simpler numerical model in which there is no need to capture the embedded discontinuities via the cohesive element or enrichment function. However, as explained previously in Wei and Anand [144], one must be cautious that representing strong discontinuities with regularized interfaces might lead to an unrealistic large volume of a fraction of grain-boundary regions if the thickness of the regularized interface is too large. The setup of the boundary value problem is depicted in Fig. 12, the grain boundary locates on the diagonal of the domain, which is separated into two grains having different orientations $\left(30^{\circ}\right.$ and $\left.60^{\circ}\right)$. This pre-existing crack is purposely put inside the gain such that the intragrain crack may propagate toward the grain boundary.

We adopt the numerical values of fracture energies reported by Tromans and Meech [128], which estimates ideal fracture energies of both grain and grain boundary of a variety of minerals including halite. In this paper, the grain boundary fracture energy was estimated to $86 \%$ of the grain fracture energy: $1.155 \mathrm{~J} / \mathrm{m}^{2}$ for the grain, and 0.993 $\mathrm{J} / \mathrm{m}^{2}$ for the grain boundary, respectively. We further assume the critical resolved stress $\left(\tau_{Y}=7.5 \mathrm{MPa}\right)$, hardening parameter $(h=5 \mathrm{GPa})$, and activation energy $(Q=10.0 \mathrm{kcal} / \mathrm{mol} / \mathrm{K})$. To analyze the relationship between the crack patterns and the grain boundary fracture energy, we conduct multiple simulations where the fracture energy of the grain boundary is assumed to be $86 \%, 75 \%$, and $50 \%$ of the intra-grain counterpart. Other material properties of the grain boundary, that may differ from the grain due to diverse reasons, such as impurity, solid mass exchange, precipitation creep, etc., are out of the scope in this study but will be considered in the future. The material parameters used in the simulations can be found in Table 1. As discussed in Appendix A, the material parameters of halite are highly sensitive to thermal and mechanical conditions. The well-designed experiments, therefore, need to be conducted to calibrate the numerical models for particular engineering application purposes.

The simulated crack patterns of a bicrystal with three different grain-boundary fracture energies $(86 \%, 75 \%$, and $50 \%$ of intragrain fracture energy), are shown in Fig. 13. In particular, the damage represented by the combined phase field (the left figures) and the magnitude of the plastic slip (the right figures) for the cases where the grain-boundary fracture energy is $86 \%, 75 \%$ and $50 \%$ are (a) and (b), (c) and (d), and (e) and (f) accordingly. In the 86\% case, the crack initiates from the pre-existing crack tip and propagates through the grain boundary until reaching the right end of the domain. In other words, the grain boundary causes the refraction of the crack, a phenomenon commonly observed in materials with inter-layer(s) [145].

When the grain-boundary fracture energy is reduced to $70 \%$ of the grain fracture energy, as shown in Fig. 13(c) and (d), the main fracture behavior is similar to the previous case before reaching the grain boundary. However, when the crack reaches the grain boundary, it grows along the grain boundary before the crack refraction occurs. In the last case where the grain-boundary fracture energy is further reduced to $50 \%$ of the grain fracture energy, the crack simply propagates along the grain boundary once reaching it. One interesting implication of this result is that the assumption that cracks only occur along the grain boundary may only be valid when the interface is sufficiently weak. Otherwise, it is possible that the grain boundary may lead to crack refraction, which may in return lead to damage and even fragmentation of crystal grains. Although this example is significantly simplified set-up, this result is an indicator that 
Case 1 with the vertical strain levels of each sequence: $0.069 \%, 0.072 \%$, and $0.080 \%$
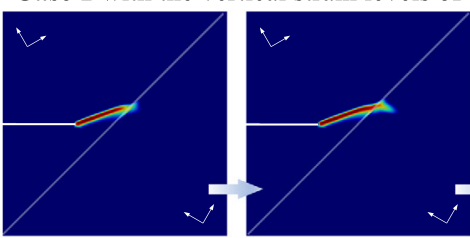

(a)

Case 2 with the vertical strain levels of each sequence: $0.068 \%, 0.073 \%$, and $0.080 \%$

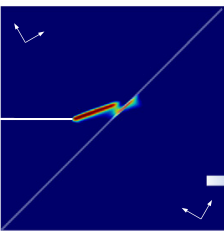

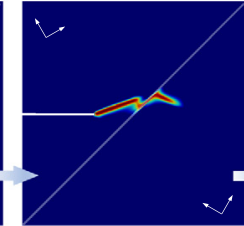

(c)
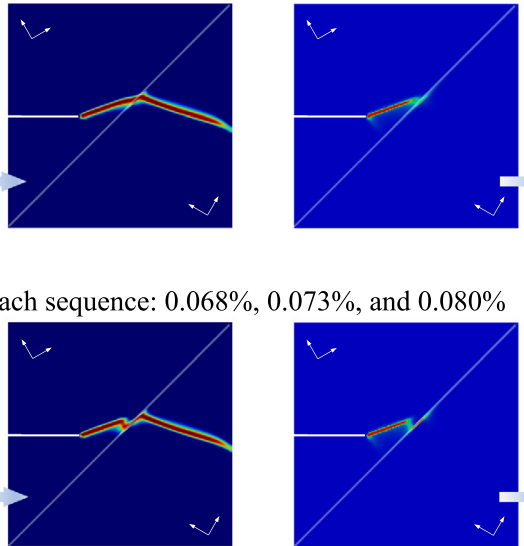

$\checkmark$

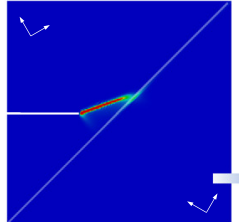

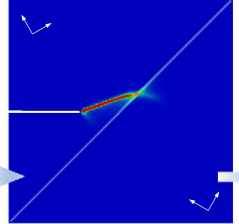

(b)
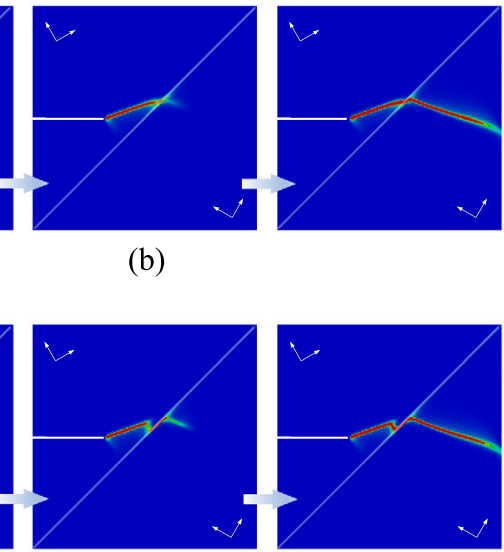

(d)

Case 3 with the vertical strain levels of each sequence: $0.063 \%, 0.066 \%$, and $0.076 \%$

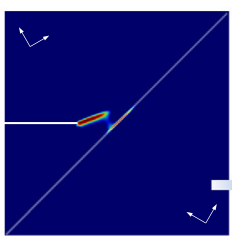

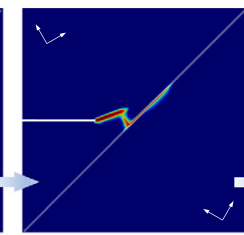

(e)

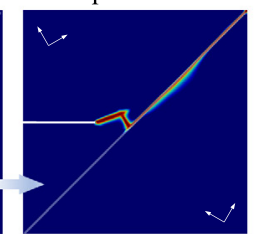

Phase Field

$\begin{array}{llll}0.7 & 0.8 & 0.9 & 1.0\end{array}$

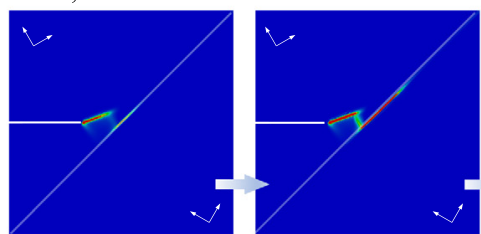

(f)

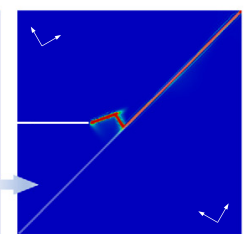

Plastic Slip

$0.0 \quad 1.0 \mathrm{E}-3 \quad 2.0 \mathrm{E}-3$

Fig. 13. Phase-field values (left) and plastic slip results (right) patterns with different fracture energies of the grain boundary are illustrated. Case 1 (a) and (b) assumes the fracture energy of the grain boundary with $86 \%$ of grain fracture energy; Case 2 (c) and (d) with $75 \%$; Case 3 (e) and (f) with $50 \%$ of the grain fracture energy, respectively.

the intragrain fractures could be significant in polycrystalline materials in certain circumstance. In those cases, the phase field approach may provide a convenient means to capture the interactions of intragrain and grain-boundary fractures.

\section{Conclusions}

In this work, we develop a framework that combines the multi-phase-field method and the crystal plasticity theory under non-isothermal conditions for modeling single-crystal halite. This work is a starting point for modeling coupled thermo-hydro-mechanical behavior of polycrystalline rock salt. Under the postulation of microforces associated with plastic and fracture behaviors, the microstructure information coming from its slip system is incorporated into the governing equations. Both rate-dependent and rate-independent features are captured via the crystal plasticity stress update algorithm. Besides, the multi-phase-field method presents anisotropic damage of which each directional crack phase-field is associated with the slip planes. The energy balance equation is further encapsulated into the numerical framework to address the heat conduction, plastic dissipation, and thermoelastic structural heating. The numerical examples for single crystals demonstrate that, under the mechanical loading, the proposed numerical framework well captures the material anisotropy by rotating the slip-system orientations and the variation of inelastic to fracture behavior under different temperature conditions. Furthermore, the thermal loading by heat flux in a single crystal exhibits the anisotropic deformation, or distortion due to temperature increase. The tension test by changing the loading rate indicates the brittle-to-ductile transition along with the change of fracture patterns. Finally, the numerical example using a bicrystal presents the impact of fracture energy of the grain boundary on the inter- and intra-granular fracture, in which the grain boundary is simplified using a single cleavage crystal material. Although this framework for single-crystal halite is a starting point to be utilized as field scale simulations for polycrystalline rock salt, the results presented here indicate that this contribution provides qualitatively compatible physical behaviors of halite and 
potential applicability for practical engineering applications, e.g., a geologic repository for heating generating nuclear waste.

\section{Acknowledgments}

The first author's effort is supported by the nuclear energy university program from department of energy under grant contract DE-NE0008534, while effort of the second author is supported by the Earth Materials and Processes program from the US Army Research Office under grant contract W911NF-15-1-0581, the Dynamic Materials and Interactions Program from the Air Force Office of Scientific Research under grant contract FA9550-17-1-0169, and the Mechanics of Materials and Structure program at National Science Foundation under grant contract CMMI-1462760. These supports are gratefully acknowledged. The authors would also like to thank their colleagues at Sandia National Laboratories, Drs. Kristopher Kuhlman, Hongkyu Yoon, and Mathew Ingraham, and their former research group member, Professor Jinhyun Choo of Hong Kong University for fruitful discussion. The three anonymous reviewers have provided many constructive suggestions and insightful feedback, which are gratefully acknowledged.

\section{Appendix A. Stress-point simulations for model calibration}

In this section, we perform the material parameter calibration using the previous experimental data for singlecrystal halite (e.g., Carter and Heard [146]). Furthermore, we identify the significant sensitivity of material parameters for halite and indicate the limitation of the proposed numerical model and future direction toward modeling of polycrystalline rock salt.

As stated previously, the material properties of halite or rock salt significantly depend on the environmental (e.g., temperature) and loading conditions (e.g., loading rate). As such, identifying the material properties of halite itself is an integral part of engineering applications. Furthermore, calibrating numerical models to capture a wide spectrum of behaviors of halite under different conditions is a challenging task. As a first step toward calibration for polycrystalline rock salt, we used the previously conducted experimental works by Carter and Heard [146] to investigate how the proposed model captures the actual experimental work and evaluate the identified material properties of single-crystal halite. The calibration leverages the open source software toolkit Dakota [147], in which the gradient-based least square algorithm (NL2SOL) is used. This method minimizes the residual function, and here we chose differential stress at each loading step to constitute the objective function, and use a gradient-based optimization algorithm to identify the material parameters. The parameters for calibration include the bulk modulus $(K)$, critical resolved shear strength $\left(\tau_{Y}\right)$, hardening parameter $(h)$, activation energy $(Q)$, shape factor $\left(C_{0}\right)$, and stress exponent $(p)$. Note that Carter and Heard [146] used artificially grown single-crystal halite, but the preparation of the sample lacked the details such as the amount of the impurity, which may significantly influence the material properties. The further information can be found from Carter and Heard [146]. Furthermore, the information for elastic behavior within the small deformation regime was not clearly reported. However, the experimential data of single-crystal halite is adopted for a preliminary calibration testing.

We first choose 8 sets of test results, which include two loading rates $\left(1.0 \times 10^{-4} / \mathrm{s}\right.$ and $\left.1.0 \times 10^{-7} / \mathrm{s}\right)$ with four temperature conditions $\left(25,100,200\right.$, and $\left.300{ }^{\circ} \mathrm{C}\right)$ with the vertical strain up to $10 \%$. Note that the proposed numerical model for stress point simulations may not be acceptable to capture these ranges of test conditions including large strain loading. However, we believe this process leads us to check the potential applicability of the proposed model and its limitation. As can be seen in Fig. 14, the overall behavior of the proposed model is observed to be similar to the mechanical behavior of halite obtained from the experiment. However, the calibration analysis did not reach its (relative) tolerance, which was set to $1.0 \times 10^{-6}$. This indicates limitations of the proposed model under these wide ranges of test conditions. Due to insufficient experimental information and severe nonlinearity within the small deformation range $(<1 \%)$, the significant errors within this region are observed, especially when the temperature was set to $25^{\circ} \mathrm{C}$. Therefore, we reduce the experimental set for calibration and focus on the limited conditions of testing. The calibrated material parameters are presented as Case 1 in Table 2.

We next streamline the experimental results and use them for stress-strain curves with two loading rates $\left(1.0 \times 10^{-4} / \mathrm{s}\right.$ and $\left.1.0 \times 10^{-7} / \mathrm{s}\right)$ with two temperature conditions $\left(25^{\circ} \mathrm{C}, 100^{\circ} \mathrm{C}\right)$. Furthermore, we analyze the inverse problem within the small deformation regime $(<2.0 \%)$. As a result, we observe the improved calibration results as in Fig. 15 for the small deformation with temperature conditions of 25 and $100{ }^{\circ} \mathrm{C}$. The initial strain region, less than $0.2 \%$ of vertical strain, is still not calibrated enough due to the lack of information, whereas the calibrated behavior 


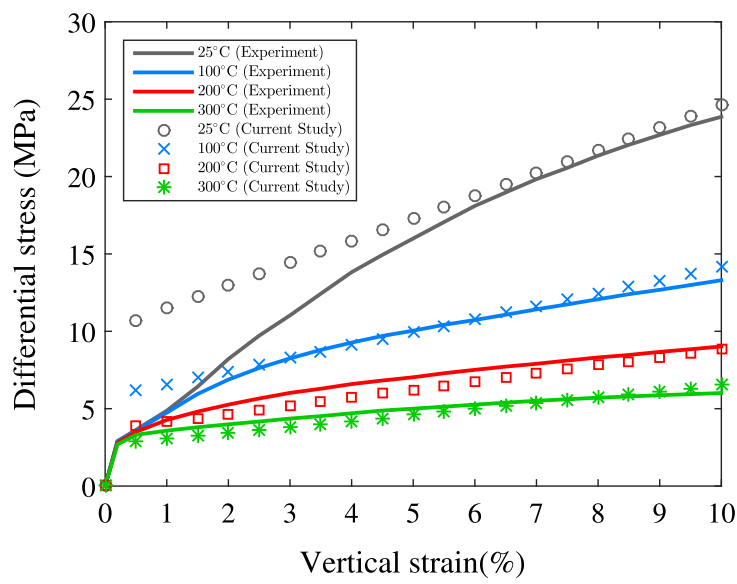

(a) Strain rate $1.0 \times 10^{-4} / \mathrm{s}$.

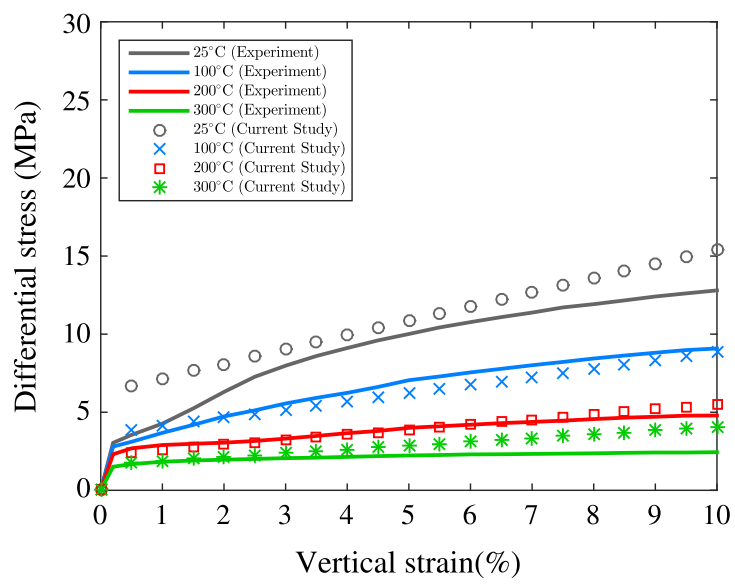

(b) Strain rate $1.0 \times 10^{-7} / \mathrm{s}$.

Fig. 14. Comparison of stress-strain curves from the experiment [146] and current study under different temperature $\left(25,100,200\right.$, and $\left.300^{\circ} \mathrm{C}\right)$ and strain rate conditions, (a) with the strain rate $1.0 \times 10^{-4} / \mathrm{s}$, (b) the strain rate $1.0 \times 10^{-7} / \mathrm{s}$.

Table 2

Two sets of calibrated material parameters obtained from the inverse problem using stress-point simulations.

\begin{tabular}{lllllll}
\hline & $K(\mathrm{GPa})$ & $\tau_{Y}(\mathrm{GPa})$ & $h(\mathrm{GPa})$ & $Q(\mathrm{kcal} / \mathrm{mol})$ & $C_{0}$ & 0.1 \\
Case 1 & 25.0 & 0.9 & 6.6 & 24.0 & 15.0 \\
Case 2 & 1.1 & 1.9 & 80.1 & 8.0 & 1.0 & 10.0 \\
\hline
\end{tabular}

of halite is well matched with the experimental results. The calibrated material parameters are presented as Case 2 in Table 2. Compared to Case 1, the values of calibrated parameters are significantly changed including the bulk modulus $K$, hardening parameter $h$, and activation energy $Q$.

This analysis further indicates that, although the proposed model may not be suitable for a wide range of testing conditions, we can still utilize this for a specific purpose. This conclusion not only leaves us room to improve the numerical model but indicates the future direction of our research for polycrystalline rock salt which includes very complicated thermo-hydro-mechanical couplings. By combining the well designed experimental work for a geological repository stability, we may utilize material identification and calibration process to better capture the actual stability analysis. Note that the current calibration did not include the effect of damage. The calibration will be further expanded to actual sample considering damage in future study.

\section{Appendix B. Benchmark: multiple phase-field method for anisotropic crack propagation}

This section describes how the multi-phase-field method captures the anisotropic crack propagation. The approach for anisotropic fracture using the elastic material has been proposed by Nguyen et al. [54,55], in which the derivation and the applications are well explained. Here we adopted this idea from Nguyen et al. [54,55] and conducted a benchmark simulation to check the implementation and ensure the ability to replicate the anisotropic crack propagation in the absence of crystal plasticity. Therefore, the boundary value problem from Nguyen et al. [54] is reused as a benchmark. For more details on other alternative higher-order single-phase-field approaches that model the anisotropic fracture with strong anisotropic surface energy, we refer readers to Li et al. [57], Teichtmeister et al. [58].

The test set-up for a guided crack propagation is described in Fig. 16(a). The square domain with the length $L$ is $1.0 \mathrm{~mm}$. Note that the higher fracture toughness is assigned to the top and bottom parts of the domain outside the guided line to prevent any crack propagation outside the guided line. For directional fracture, two phase-field variables are used with the cleavage planes having normal vectors $135^{\circ}$ and $45^{\circ}$ directions, respectively. Note that the material is assumed to be elastic with the bulk modulus of $175 \mathrm{GPa}$, Poisson's ratio of 0.3 , fracture energy of $2.7 \mathrm{~kJ} / \mathrm{m}^{2}$, the length scale of $0.01 \mathrm{~mm}$, and the anisotropy factor $\beta$ of 50. As can be seen in Fig. 16(c) and (d), the 


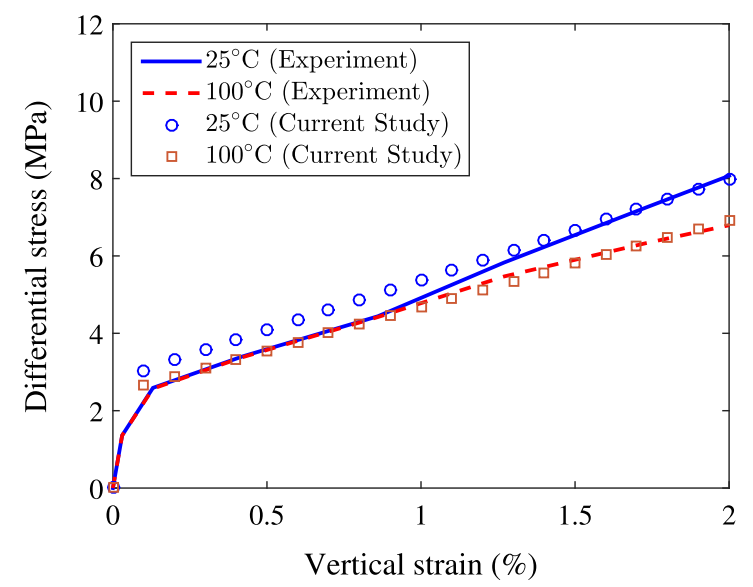

(a) Strain rate $1.0 \times 10^{-4} / \mathrm{s}$.

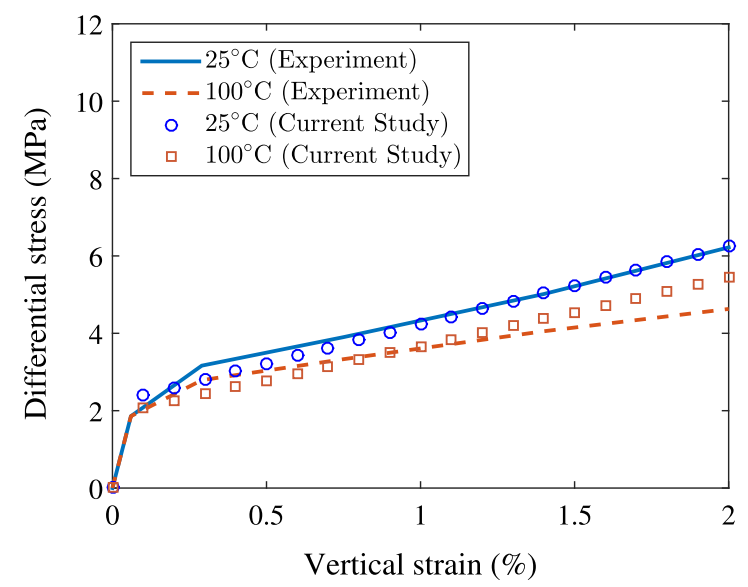

(b) Strain rate $1.0 \times 10^{-7} / \mathrm{s}$.

Fig. 15. Comparison of stress-strain curves from the experiment [146] and current study under different temperature $\left(25\right.$ and $\left.100{ }^{\circ} \mathrm{C}\right)$ and strain rate conditions, (a) with the strain rate $1.0 \times 10^{-4} / \mathrm{s}$, (b) the strain rate $1.0 \times 10^{-7} / \mathrm{s}$.

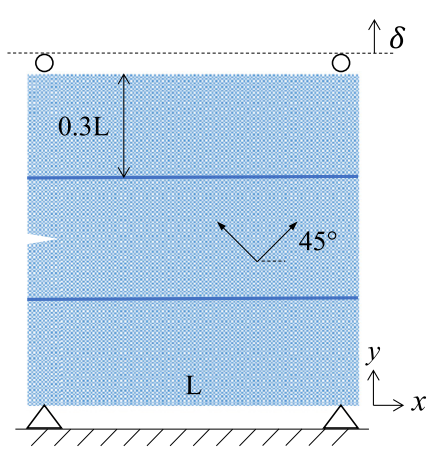

(a) Test set-up.
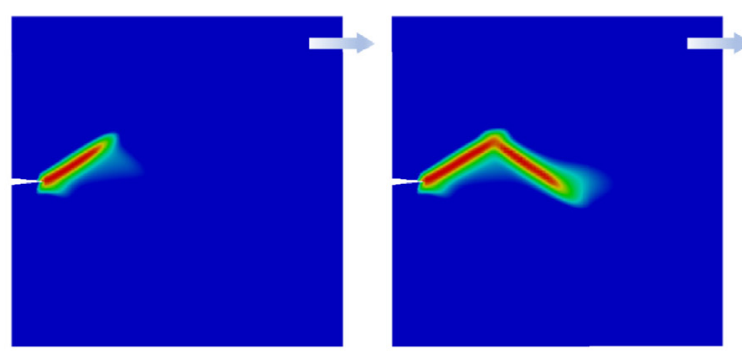

(b) Combined Crack Phase Field $\left(d_{e q}=d_{1}+d_{2}-d_{1} d_{2}\right)$.

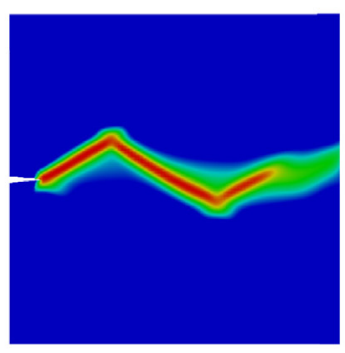

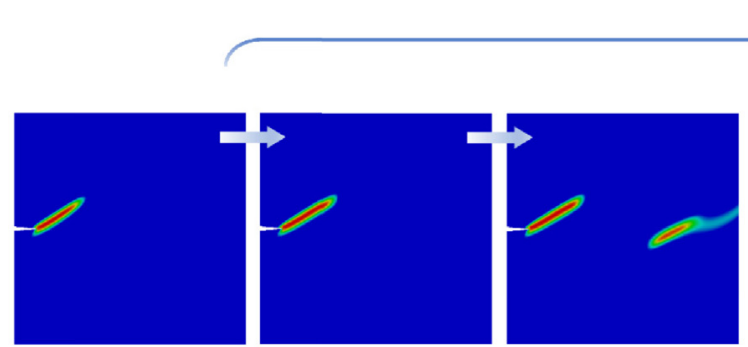

(c) Phase Field 1.
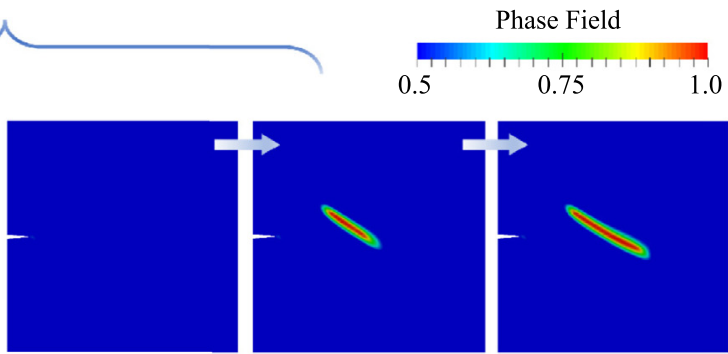

(d) Phase Field 2.

Fig. 16. The concept of multiple phase-field method for anisotropic crack propagation, (a) test set-up for guided crack propagation (the top and bottom parts of the domain outside the guided line have higher fracture toughness not to initiate cracks, (b) the combined crack phase field using the equation suggested by Nguyen et al. [54,55], (c) the crack propagation of phase-field 1, (d) the crack propagation of phase-field 2.

phase field 1 starts to grow from the edge of the existing crack while the phase field 2 does not grow until the crack hits the guided line. Then, the crack captured by phase field 2 begins to propagate until it hits the bottom guided line. This process continues and the crack represented by the combined phase fields exhibits a zig-zag pattern as shown in Fig. 16. The combined crack phase field, or the equivalent phase field, is calculated using the given equation which is closely related to the degradation function for multiple phase-field method. The detailed theoretical bases, derivations of the anisotropic surface energy plots can be further found in Nguyen et al. [54,55]. Our new contribution is twofold. 
First, we combine this multi-phase-field method with the crystal plasticity constitutive models for single-crystal grain. Second, we extend this damage-plasticity model for capturing the thermo-mechanics of crystalline rock.

\section{References}

[1] K.L. Kuhlman, B. Malama, Brine Flow in Heated Geologic Salt (SAND2013-1944). Technical Report, Sandia National Labs., Albuquerque, NM (USA), 2013.

[2] T. von Berlepsch, B. Haverkamp, Salt as a host rock for the geological repository for nuclear waste, Elements 12 (4) (2016) $257-262$.

[3] A. Urquhart, S. Bauer, Experimental determination of single-crystal halite thermal conductivity, diffusivity and specific heat from $75^{\circ} \mathrm{C}$ to $300{ }^{\circ}$ C, Int. J. Rock Mech. Min. Sci. 78 (2015) 350-352.

[4] W. Bechthold, E. Smailos, S. Heusermann, W. Bollingerfehr, B. Bazargan Sabet, T. Rothfuchs, P. Kamlot, J. Grupa, S. Olivella, F.D. Hansen, Backfilling and sealing of underground repositories for radioactive waste in salt (BAMBUS-II Project), in: Commission of the European Communities, EUR, Vol. 20621, 2004.

[5] K.S. Chan, S.R. Bodner, D.E. Munson, Recovery and healing of damage in WIPP salt, Int. J. Damage Mech. 7 (2) (1998) $143-166$.

[6] F.D. Hansen, C.D. Leigh, Salt Disposal of Heat-Generating Nuclear Waste (SAND2011-0161), Sandia National Laboratories, Albuquerque, NM, 2011.

[7] R.L. Bradshaw, F. Sanchez, Migration of brine cavities in rock salt, J. Geophys. Res. 74 (17) (1969) 4209-4212.

[8] E.J. Nowak, D.F. McTigue, Interim Results of Brine Transport Studies in the Waste Isolation Pilot Plant (WIPP) (SAND-87-0880). Tehnical Report, Sandia National Labs., Albuquerque, NM (USA), 1987.

[9] R.L. Beauheim, S.M. Howarth, P. Vaughn, S.W. Webb, K.W. Larson, Integrated Modeling and Experimental Programs to Predict Brine and Gas Flow at the Waste Isolation Pilot Plant (No. SAND-94-0599C; CONF-941053-1). Technical Report, Sandia National Labs., Albuquerque, NM (United States), 1995.

[10] Z. Schléder, J.L. Urai, S. Nollet, C. Hilgers, Solution-precipitation creep and fluid flow in halite: a case study of Zechstein (Z1) rocksalt from Neuhof salt mine (Germany), Int. J. Earth Sci. 97 (5) (2008) 1045-1056.

[11] F.D. Hansen, S.J. Bauer, S.T. Broome, G.D. Callahan, Coupled Thermal-Hydrological-Mechanical Processes in Salt: Hot Granular Salt Consolidation, Constitutive Model and Micromechanics (SAND2012-9893P). Technical Report, Sandia National Labs., Albuquerque, NM (United States), 2012.

[12] M. Guillope, J.P. Poirier, Dynamic recrystallization during creep of single-crystalline halite: An experimental study, J. Geophys. Res. 84 (B10) (1979) 5557-5567.

[13] N.L Carter, F.D. Hansen, P.E. Senseny, Stress magnitudes in natural rock salt, J. Geophys. Res. 87 (B11) (1982) 9289-9300.

[14] N.L. Carter, F.D. Hansen, Creep of rocksalt, Tectonophysics 92 (4) (1983) 275-333.

[15] J.L. Urai, C.J. Spiers, H.J. Zwart, G.S. Lister, Weakening of rock salt by water during long-term creep, Nature 324 (6097) (1986) $554-557$.

[16] N.L. Carter, S.T. Horseman, J.E. Russell, J. Handin, Rheology of rocksalt, J. Struct. Geol. 15 (9-10) (1993) $1257-1271$.

[17] P.E. Senseny, F.D. Hansen, J.E. Russell, N.L. Carter, J.W. Handin, Mechanical behaviour of rock salt: phenomenology and micromechanisms, Int. J. Rock Mech. Min. Sci. Geomech. Abstr. 29 (4) (1992) 363-378.

[18] J.L. Urai, Z. Schléder, C.J. Spiers, P.A. Kukla, Flow and transport properties of salt rocks, Dyn. Complex Intracont. Basins: Central Eur. Basin Syst. (2008) 277-290.

[19] F.D. Hansen, et al., Micromechanics of isochoric salt deformation, in: 48th US Rock Mechanics/Geomechanics Symposium, American Rock Mechanics Association, 2014.

[20] D.E. Munson, P.R. Dawson, Constitutive Model for the Low Temperature Creep of Salt (With Application to WIPP) (SAND-79-1853). Technical Report, Sandia National Labs., Albuquerque, NM (USA), 1979.

[21] K.S. Chan, A.F. Fossum, S.R. Bodner, D.E. Munson, et al., Constitutive representation of damage healing in WIPP salt, in: The 35th US Symposium on Rock Mechanics (USRMS), American Rock Mechanics Association, 1995.

[22] S. Miao, M.L. Wang, H.L. Schreyer, Constitutive models for healing of materials with application to compaction of crushed rock salt, J. Eng. Mech. 121 (10) (1995) 1122-1129.

[23] D.E. Munson, Constitutive model of creep in rock salt applied to underground room closure, Int. J. Rock Mech. Min. Sci. 34 (2) (1997) 233-247.

[24] M. Aubertin, M.R. Julien, S. Servant, D.E. Gill, A rate-dependent model for the ductile behavior of salt rocks, Can. Geotech. J. 36 (4) (1999) 660-674.

[25] S. Olivella, A. Gens, A constitutive model for crushed salt, Int. J. Numer. Anal. Methods Geomech. 26 (7) (2002) $719-746$.

[26] C. Zhu, C. Arson, A model of damage and healing coupling halite thermo-mechanical behavior to microstructure evolution, Geotech. Geol. Eng. 33 (2) (2015) 389-410.

[27] S.T. Broome, S.J. Bauer, F.D. Hansen, M.M. Mills, Mechanical response and microprocesses of reconsolidating crushed salt at elevated temperature, Rock Mech. Rock Eng. 48 (6) (2015) 2615-2629.

[28] X. Shen, C. Zhu, C. Arson, et al., Chemo-mechanical damage and healing of granular salt: micro-macro modeling, in: 50th US Rock Mechanics/Geomechanics Symposium, American Rock Mechanics Association, 2016.

[29] S. Olivella, J. Carrera, A. Gens, E.E. Alonso, Nonisothermal multiphase flow of brine and gas through saline media, Transp. Porous Media 15 (3) (1994) 271-293.

[30] S. Olivella, J. Carrera, A. Gens, E.E. Alonso, Porosity variations in saline media caused by temperature gradients coupled to multiphase flow and dissolution/precipitation, Transp. Porous Media 25 (1) (1996) 1-25.

[31] S. Olivella, A. Gens, J. Carrera, E.E. Alonso, Numerical formulation for a simulator (CODE_BRIGHT) for the coupled analysis of saline media, Eng. Comput. 13 (7) (1996) 87-112. 
[32] S. Olivella, A. Gens, Vapour transport in low permeability unsaturated soils with capillary effects, Transp. Porous Media 40 (2) (2000) 219-241.

[33] J.R. Rice, Inelastic constitutive relations for solids: an internal-variable theory and its application to metal plasticity, J. Mech. Phys. Solids 19 (6) (1971) 433-455.

[34] D. Peirce, R.J. Asaro, A. Needleman, An analysis of nonuniform and localized deformation in ductile single crystals, Acta Metall. 30 (6) (1982) 1087-1119.

[35] K.S. Havner, Finite Plastic Deformation of Crystalline Solids, Cambridge University Press, 1992.

[36] M.E. Gurtin, E. Fried, L. Anand, The Mechanics and Thermodynamics of Continua, Cambridge University Press, 2010.

[37] E.A. de Souza Neto, D. Peric, D.R.J. Owen, Computational Methods for Plasticity: Theory and Applications, John Wiley \& Sons, 2011.

[38] A.M. Cuitino, M. Ortiz, Computational modelling of single crystals, Modelling Simulation Mater. Sci. Eng. 1 (3) (1993) 225.

[39] R.I. Borja, J.R. Wren, Discrete micromechanics of elastoplastic crystals, Internat. J. Numer. Methods Engrg. 36 (22) (1993) $3815-3840$.

[40] L. Anand, M. Kothari, A computational procedure for rate-independent crystal plasticity, J. Mech. Phys. Solids 44 (4) (1996) 525-558.

[41] C. Miehe, J. Schröder, A comparative study of stress update algorithms for rate-independent and rate-dependent crystal plasticity, Internat. J. Numer. Methods Engrg. 50 (2) (2001) 273-298.

[42] R.I. Borja, H. Rahmani, Computational aspects of elasto-plastic deformation in polycrystalline solids, J. Appl. Mech. 79 (3) (2012) 031024.

[43] M. Tjioe, R.I. Borja, On the pore-scale mechanisms leading to brittle and ductile deformation behavior of crystalline rocks, Int. J. Numer. Anal. Methods Geomech. 39 (11) (2015) 1165-1187.

[44] C. Miehe, F. Welschinger, M. Hofacker, Thermodynamically consistent phase-field models of fracture: Variational principles and multi-field FE implementations, Internat. J. Numer. Methods Engrg. 83 (10) (2010) 1273-1311.

[45] C. Miehe, M. Hofacker, F. Welschinger, A phase field model for rate-independent crack propagation: Robust algorithmic implementation based on operator splits, Comput. Methods Appl. Mech. Engrg. 199 (45) (2010) 2765-2778.

[46] J.D. Clayton, J. Knap, Phase field modeling of twinning in indentation of transparent crystals, Modelling Simulation Mater. Sci. Eng. 19 (8) (2011) 085005.

[47] J.D. Clayton, J. Knap, Phase field modeling of directional fracture in anisotropic polycrystals, Comput. Mater. Sci. 98 (2015) $158-169$.

[48] S. Na, W. Sun, M.D. Ingraham, H. Yoon, Effects of spatial heterogeneity and material anisotropy on the fracture pattern and macroscopic effective toughness of mancos shale in brazilian tests, J. Geophys. Res. (2017).

[49] O. Aslan, N.M. Cordero, A. Gaubert, S. Forest, Micromorphic approach to single crystal plasticity and damage, Internat. J. Engrg. Sci. 49 (12) (2011) 1311-1325.

[50] J.D. Clayton, Deformation, fracture, and fragmentation in brittle geologic solids, Int. J. Fract. 163 (1) (2010) 151-172.

[51] M. Koslowski, M. Ortiz, A multi-phase field model of planar dislocation networks, Modelling Simulation Mater. Sci. Eng. 12 (6) (2004) 1087.

[52] T. Takaki, Y. Hisakuni, T. Hirouchi, A. Yamanaka, Y. Tomita, Multi-phase-field simulations for dynamic recrystallization, Comput. Mater. Sci. 45 (4) (2009) 881-888.

[53] K. Oshima, T. Takaki, M. Muramatsu, Development of multi-phase-field crack model for crack propagation in polycrystal, Int. J. Comput. Mater. Sci. Eng. 3 (02) (2014) 1450009.

[54] T.T. Nguyen, J. Réthoré, M.C. Baietto, Phase field modelling of anisotropic crack propagation, Eur. J. Mech. A (2017).

[55] T.T. Nguyen, J. Réthoré, J. Yvonnet, M.C. Baietto, Multi-phase-field modeling of anisotropic crack propagation for polycrystalline materials, Comput. Mech. (2017) 1-26.

[56] I. Steinbach, Phase-field models in materials science, Model. Simul. Mater. Sci. Eng. 17 (7) (2009) 073001.

[57] B. Li, C. Peco, D. Millán, I. Arias, M. Arroyo, Phase-field modeling and simulation of fracture in brittle materials with strongly anisotropic surface energy, Internat. J. Numer. Methods Engrg. 102 (3-4) (2015) 711-727.

[58] S. Teichtmeister, D. Kienle, F. Aldakheel, M.A. Keip, Phase field modeling of fracture in anisotropic brittle solids, Int. J. Non-Linear Mech 97 (2017) 1-21.

[59] M. Ekh, R. Lillbacka, K. Runesson, A model framework for anisotropic damage coupled to crystal (visco) plasticity, Int. J. Plast. 20 (12) (2004) 2143-2159.

[60] R. de Borst, C.V. Verhoosel, Gradient damage vs phase-field approaches for fracture: Similarities and differences, Comput. Methods Appl. Mech. Engrg. 312 (2016) 78-94.

[61] J. Choo, W. Sun, Coupled phase-field and plasticity modeling of geological materials: From brittle fracture to ductile flow, Comput. Methods Appl. Mech. Engrg. (2017).

[62] U. Lee, G.A. Lesieutre, L. Fang, Anisotropic damage mechanics based on strain energy equivalence and equivalent elliptical microcracks, Int. J. Solids Struct. 34 (33-34) (1997) 4377-4397.

[63] J.C. Simo, J.W. Ju, On continuum damage-elastoplasticity at finite strains, Comput. Mech. 5 (5) (1989) 375-400.

[64] B. Budiansky, T.T. Wu, Theoretical Prediction of Plastic Strains of Polycrystals. Technical Report, Havard University Cambridge Mass, 1961.

[65] R.I. Borja, Plasticity: Modeling \& Computation, Springer Science \& Business Media, 2013.

[66] J.H. Williams, Crystal Engineering: How Molecules Build Solids, Morgan \& Claypool Publishers, 2017.

[67] C.B. Carter, M.G. Norton, Ceramic Materials: Science and Engineering, Springer Science \& Business Media, 2007.

[68] W.D. Callister Jr., D.G. Rethwisch, Fundamentals of Materials Science and Engineering: An Integrated Approach, John Wiley \& Sons, 2012.

[69] W.G. Moffat, G.W. Pearsall, J. Wulff, The Structure and Properties of Materials. Volume 1, Structure, Vol. 1, Wiley, New York, 1964.

[70] F. Aldakheel, Micromorphic approach for gradient-extended thermo-elastic-plastic solids in the logarithmic strain space, Contin. Mech. Thermodyn. (2017) 1-11. 
[71] C. Miehe, M. Hofacker, L.M. Schaenzel, F. Aldakheel, Phase field modeling of fracture in multi-physics problems. Part II. Coupled brittleto-ductile failure criteria and crack propagation in thermo-elastic-plastic solids, Comput. Methods Appl. Mech. Engrg. 294 (2015) $486-522$.

[72] L. Anand, M.E. Gurtin, Thermal effects in the superelasticity of crystalline shape-memory materials, J. Mech. Phys. Solids 51 (6) (2003) 1015-1058.

[73] J.D. Clayton, Nonlinear Mechanics of Crystals, Vol. 77, Springer Science \& Business Media, 2010.

[74] F.T. Meissonnier, E.P. Busso, N.P. O’Dowd, Finite element implementation of a generalised non-local rate-dependent crystallographic formulation for finite strains, Int. J. Plast. 17 (4) (2001) 601-640.

[75] B. Bourdin, G.A. Francfort, J.J. Marigo, The variational approach to fracture, J. Elasticity 91 (1) (2008) 5-148.

[76] B. Schmidt, F. Fraternali, M. Ortiz, Eigenfracture: an eigendeformation approach to variational fracture, Multiscale Model. Simul. 7 (3) (2009) 1237-1266.

[77] K. Wang, W. Sun, A unified variational eigen-erosion framework for interacting brittle fractures and compaction bands in fluid-infiltrating porous media, Comput. Methods Appl. Mech. Engrg. 318 (2017) 1-32.

[78] L. De Lorenzis, A. McBride, B.D. Reddy, Phase-field modelling of fracture in single crystal plasticity, GAMM-Mitt. 39 (1) (2016) 7-34.

[79] J.D. Bredehoeft, A.W. England, D.B. Stewart, N.J. Trask, I.J. Winograd, Geologic Disposal of High-Level Radioactive Wastes: Earth-Science Perspectives, US Department of the Interior, US Geological Survey, 1978.

[80] M.E. Gurtin, Generalized Ginzburg-Landau and Cahn-Hilliard equations based on a microforce balance, Physica D 92 (3-4) (1996) 178-192.

[81] M.J. Borden, Isogeometric Analysis of Phase-Field Models for Dynamic Brittle and Ductile Fracture (Ph.D. thesis), 2012.

[82] A. Mota, W. Sun, J.T. Ostien, J.W. Foulk, K.N. Long, Lie-group interpolation and variational recovery for internal variables, Comput. Mech. (2013) 1-19.

[83] L. Stainier, M. Ortiz, Study and validation of a variational theory of thermo-mechanical coupling in finite visco-plasticity, Int. J. Solids Struct. 47 (5) (2010) 705-715.

[84] Q. Yang, L. Stainier, M. Ortiz, A variational formulation of the coupled thermo-mechanical boundary-value problem for general dissipative solids, J. Mech. Phys. Solids 54 (2) (2006) 401-424.

[85] J.C. Simo, C. Miehe, Associative coupled thermoplasticity at finite strains: formulation, numerical analysis and implementation, Comput. Methods Appl. Mech. Engrg. 98 (1) (1992) 41-104.

[86] R. Abeyaratne, S.J. Kim, J.K. Knowles, A one-dimensional continuum model for shape-memory alloys, Int. J. Solids Struct. 31 (16) (1994) 2229-2249.

[87] V.A. Lubarda, On thermodynamic potentials in linear thermoelasticity, Int. J. Solids Struct. 41 (26) (2004) 7377-7398.

[88] M. Peigney, J.P. Seguin, An incremental variational approach to coupled thermo-mechanical problems in anelastic solids. Application to shape-memory alloys, Int. J. Solids Struct. 50 (24) (2013) 4043-4054.

[89] T. Heister, M.F. Wheeler, T. Wick, A primal-dual active set method and predictor-corrector mesh adaptivity for computing fracture propagation using a phase-field approach, Comput. Methods Appl. Mech. Engrg. 290 (2015) 466-495.

[90] M. Ambati, T. Gerasimov, L. De Lorenzis, Phase-field modeling of ductile fracture, Comput. Mech. 55 (5) (2015) $1017-1040$.

[91] M.J. Borden, T.J.R. Hughes, C.M. Landis, A. Anvari, I.J. Lee, A phase-field formulation for fracture in ductile materials: Finite deformation balance law derivation, plastic degradation, and stress triaxiality effects, Comput. Methods Appl. Mech. Engrg. 312 (2016) $130-166$.

[92] A. Bartels, T. Bartel, M. Canadija, J. Mosler, On the thermomechanical coupling in dissipative materials: a variational approach for generalized standard materials, J. Mech. Phys. Solids 82 (2015) 218-234.

[93] M. Canadija, J. Mosler, On the thermomechanical coupling in finite strain plasticity theory with non-linear kinematic hardening by means of incremental energy minimization, Int. J. Solids Struct. 48 (7) (2011) 1120-1129.

[94] J. Lemaitre, How to use damage mechanics, Nucl. Eng. Des. 80 (2) (1984) 233-245.

[95] J.C. Simo, J.W. Ju, Strain-and stress-based continuum damage models-II. Computational aspects, Int. J. Solids Struct. 23 (7) (1987) $841-869$.

[96] J.W. Ju, On energy-based coupled elastoplastic damage theories: constitutive modeling and computational aspects, Int. J. Solids Struct. 25 (7) (1989) 803-833.

[97] R. de Borst, J. Pamin, M.G.D. Geers, On coupled gradient-dependent plasticity and damage theories with view to localization analysis, Eur. J. Mech. A 18 (6) (1999) 939-962.

[98] O. Coussy, Poromechanics, John Wiley \& Sons, 2004.

[99] W. Sun, A unified method to predict diffuse and localized instabilities in sands, Geomech. Geoeng. 8 (2) (2013) 65-75.

[100] W. Sun, J.T. Ostien, A.G. Salinger, A stabilized assumed deformation gradient finite element formulation for strongly coupled poromechanical simulations at finite strain, Int. J. Numer. Anal. Methods Geomech. 37 (16) (2013) 2755-2788.

[101] W. Sun, A stabilized finite element formulation for monolithic thermo-hydro-mechanical simulations at finite strain, Internat. J. Numer. Methods Engrg. 103 (11) (2015) 798-839.

[102] K. Wang, W. Sun, Anisotropy of a tensorial bishop's coefficient for wetted granular materials, J. Eng. Mech. (2015) B4015004.

[103] S. Na, W. Sun, Wave propagation and strain localization in a fully saturated softening porous medium under the non-isothermal conditions, Int. J. Numer. Anal. Methods Geomech. 40 (10) (2016) 1485-1510. http://dx.doi.org/10.1002/nag.2505.

[104] S. Na, W. Sun, Computational thermo-hydro-mechanics for multiphase freezing and thawing porous media in the finite deformation range, Comput. Methods Appl. Mech. Engrg. 318 (2017) 667-700.

[105] J. Choo, R.I. Borja, Stabilized mixed finite elements for deformable porous media with double porosity, Comput. Methods Appl. Mech. Engrg. 293 (2015) 131-154.

[106] J. Choo, J.A. White, R.I. Borja, Hydromechanical modeling of unsaturated flow in double porosity media, Int. J. Geomech. 16 (6) (2016) D4016002.

[107] J.P. Hirth, J. Lothe, Theory of Dislocations, John Wiley \& Sons, 1982. 
[108] J.B. Wachtman, W.R. Cannon, M.J. Matthewson, Mechanical Properties of Ceramics, John Wiley \& Sons, 2009.

[109] M.A. Durand, The temperature variation of the elastic moduli of NaCl, KCl and MgO, Phys. Rev. 50 (5) (1936) 449.

[110] R.A. Bartels, D.E. Schuele, Pressure derivatives of the elastic constants of $\mathrm{NaCl}$ and $\mathrm{KCl}$ at $295 \mathrm{~K}$ and $195 \mathrm{~K}$, J. Phys. Chem. Solids 26 (3) (1965) 537-549.

[111] F. Birch, Finite strain isotherm and velocities for single-crystal and polycrystalline $\mathrm{NaCl}$ at high pressures and $300 \mathrm{~K}$, J. Geophys. Res. 83 (B3) (1978) 1257-1268.

[112] W.C. Oliver, G.M. Pharr, An improved technique for determining hardness and elastic modulus using load and displacement sensing indentation experiments, J. Mater. Res. 7 (6) (1992) 1564-1583.

[113] R.J. Asaro, Crystal plasticity, J. Appl. Mech. 50 (4b) (1983) 921-934.

[114] H. Amor, J.J. Marigo, C. Maurini, Regularized formulation of the variational brittle fracture with unilateral contact: numerical experiments, J. Mech. Phys. Solids 57 (8) (2009) 1209-1229.

[115] W. Qi, A. Bertram, Anisotropic continuum damage modeling for single crystals at high temperatures, Int. J. Plast. 15 (11) (1999) 1197-1215.

[116] W.R. Wawersik, D.H. Zeuch, Creep and Creep Modeling of Three Domal Salts: A Comprehensive Update (SAND-84-0568), Sandia Nationa Laboratories, 1984.

[117] Y. Liu, W. Sun, J. Fish, Determining material parameters for critical state plasticity models based on multilevel extended digital database, J. Appl. Mech. 83 (1) (2016) 011003.

[118] K. Wang, W. Sun, S. Salager, S. Na, G. Khaddour, Identifying material parameters for a micro-polar plasticity model via X-ray microcomputed tomographic (Ct) images: Lessons learned from the curve-fitting exercises, Int. J. Multiscale Comput. Eng. 14 (4) (2016).

[119] J.C. Simo, T.J.R. Hughes, Computational Inelasticity, Vol. 7, Springer Science \& Business Media, 2006.

[120] J. Kim, H.A. Tchelepi, R. Juanes, Stability, accuracy and efficiency of sequential methods for coupled flow and geomechanics, SPE J. 16 (2) (2011) 249-262.

[121] J.A. White, N. Castelletto, H.A. Tchelepi, Block-partitioned solvers for coupled poromechanics: A unified framework, Comput. Methods Appl. Mech. Engrg. 303 (2016) 55-74.

[122] K. Wang, W. Sun, A semi-implicit discrete-continuum coupling method for porous media based on the effective stress principle at finite strain, Comput. Methods Appl. Mech. Engrg. 304 (2016) 546-583.

[123] K. Wang, W. Sun, A semi-implicit micropolar discrete-to-continuum method for granular materials, in: M. Papadrakakis, V. Papadopoulos, G. Stefanou, V. Plevris (Eds.), Proceedings of European Congress on Computational Methods in Applied Science and Engineering, June, Crete Island, 2016, pp. 5-10.

[124] R.P. Pawlowski, E.T. Phipps, A.G. Salinger, Automating embedded analysis capabilities and managing software complexity in multiphysics simulation, Part I: Template-based generic programming, Sci. Program. 20 (2) (2012) 197-219.

[125] R.P. Pawlowski, E.T. Phipps, A.G. Salinger, S.J. Owen, C.M. Siefert, M.L. Staten, Automating embedded analysis capabilities and managing software complexity in multiphysics simulation, Part II: Application to partial differential equations, Sci. Program. 20 (3) (2012) $327-345$.

[126] A. Salinger, R. Bartlett, A. Bradley, Q. Chen, I. Demeshko, X. Gao, G. Hanson, A. Mota, R. Muller, E. Nielsen, et al., Albany: Using component-based design to develop a flexible, generic multiphysics analysis code, Int. J. Multiscale Comput. Eng. 14 (4) (2016) $415-438$.

[127] K. Wang, W. Sun, Data-driven discrete-continuum method for partially saturated micro-polar porous media, in: Poromechanics VI, 2017, pp. 571-578.

[128] D. Tromans, J.A. Meech, Fracture toughness and surface energies of minerals: theoretical estimates for oxides, sulphides, silicates and halides, Miner. Eng. 15 (12) (2002) 1027-1041.

[129] T.T. Nguyen, J. Yvonnet, M. Bornert, C. Chateau, K. Sab, R. Romani, R. Le Roy, On the choice of parameters in the phase field method for simulating crack initiation with experimental validation, Int. J. Fract. 197 (2) (2016) 213-226.

[130] H.R. Wenk, G. Canova, A. Molinari, H. Mecking, Texture development in halite: comparison of Taylor model and self-consistent theory, Acta Metall. 37 (7) (1989) 2017-2029.

[131] A.F. Birch, H. Clark, The thermal conductivity of rocks and its dependence upon temperature and composition, Am. J. Sci. 238 (8) (1940) $529-558$.

[132] D.D. Smith, Thermal Conductivity of Halite Using a Pulsed Laser. Technical Report, Oak Ridge Y-12 Plant, TN (USA), 1976.

[133] W. Bangerth, R. Hartmann, G. Kanschat, Deal. II—a general-purpose object-oriented finite element library, ACM Trans. Math. Softw. 33 (4) (2007) 24.

[134] W. Bangerth, T. Heister, L. Heltai, G. Kanschat, M. Kronbichler, M. Maier, B. Turcksin, T.D. Young, The deal. II library, Version 8 (2013) $1-5$.

[135] C. Burstedde, L.C. Wilcox, O. Ghattas, p4est: Scalable algorithms for parallel adaptive mesh refinement on forests of octrees, SIAM J. Sci. Comput. 33 (3) (2011) 1103-1133.

[136] M.A. Heroux, J.M. Willenbring, A new overview of the Trilinos project, Sci. Program. 20 (2) (2012) 83-88.

[137] J.A. White, R.I. Borja, Block-preconditioned Newton-Krylov solvers for fully coupled flow and geomechanics, Comput. Geosci. 15 (4) (2011) 647-659.

[138] J.A. White, R.I. Borja, Stabilized low-order finite elements for coupled solid-deformation/fluid-diffusion and their application to fault zone transients, Comput. Methods Appl. Mech. Engrg. 197 (49) (2008) 4353-4366.

[139] R.I. Borja, J. Choo, Cam-clay plasticity, Part VIII: A constitutive framework for porous materials with evolving internal structure, Comput. Methods Appl. Mech. Engrg. 309 (2016) 653-679.

[140] M.M. Rashid, S. Nemat-Nasser, A constitutive algorithm for rate-dependent crystal plasticity, Comput. Methods Appl. Mech. Engrg. 94 (2) (1992) 201-228.

[141] B. Reedlunn, Reinvestigation into Closure Predictions of Room D at the Waste Isolation Pilot Plant (SAND2016-9961). Technical Report, Sandia National Laboratories (SNL-NM), Albuquerque, NM (United States), 2016. 
[142] D.E. Munson, R.L. Jones, J.R. Ball, R.M. Clancy, D.L. Hoag, S.V. Petney, Overtest for Simulated Defense High-Level Waste (Room B): In situ Data Report (May 1984-February 1988): Waste Isolation Pilot Plant (WIPP) Thermal/Structural Interactions Program (SAND-89-2671). Technical Report, Sandia National Labs., Albuquerque, NM (USA), 1990.

[143] M.S. Paterson, T. Wong, Experimental Rock Deformation-The Brittle Field, Springer Science \&amp; Business Media, 2005.

[144] Y.J. Wei, L. Anand, Grain-boundary sliding and separation in polycrystalline metals: application to nanocrystalline fcc metals, J. Mech. Phys. Solids 52 (11) (2004) 2587-2616.

[145] K.H. Nam, I.H. Park, S.H. Ko, Patterning by controlled cracking, Nature 485 (7397) (2012) 221.

[146] N.L. Carter, H.C. Heard, Temperature and rate dependent deformation of halite, Am. J. Sci. 269 (3) (1970) 193-249.

[147] B.M. Adams, W.J. Bohnhoff, K.R. Dalbey, J.P. Eddy, M.S. Eldred, D.M. Gay, K. Haskell, P.D. Hough, L.P. Swiler, DAKOTA, A Multilevel Parallel Object-Oriented Framework for Design Optimization, Parameter Estimation, Uncertainty Quantification, and Sensitivity Analysis: Version 5.0 User's Manual. Tech. Rep. SAND2010-2183, Sandia National Laboratories, 2009. 